\title{
HYDROGEOLOGIC DATA FOR CARROLL COUNTY, ARKANSAS
}

By J.V. Brahana, Valarie A. Leidy, John Lindt, and S.A. Hodge

\section{U.S. GEOLOGICAL SURVEY}

Open-File Report 93-150

Prepared in cooperation with the

ARKANSAS SOIL AND WATER CONSERVATION COMMISSION

Little Rock, Arkansas

1993 


\title{
U.S. DEPARTMENT OF THE INTERIOR BRUCE BABBITT, Secretary
}

\author{
U.S. GEOLOGICAL SURVEY \\ Dallas L. Peck, Director
}

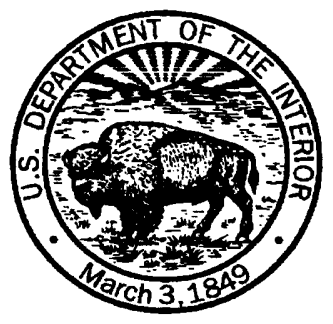

For additional information write to:

District Chief U.S. Geological Survey 2301 Federal Office Building 700 West Capitol Avenue Little Rock, Arkansas 72201
Copies of this report can be purchased from:

U.S. Geological Survey Books and Open-File Reports Section Federal Center Box 25425 Denver, Colorado 80225 


\section{CONTENTS}

Page

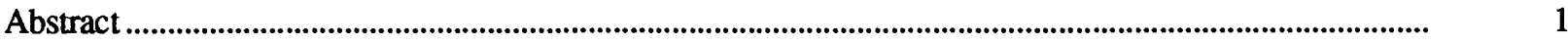

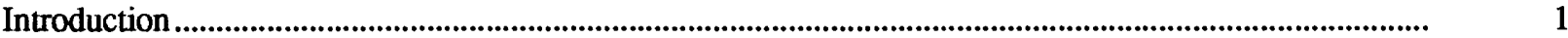

Objective and scope .....................................................................................................................

Previous investigations ....................................................................................................................

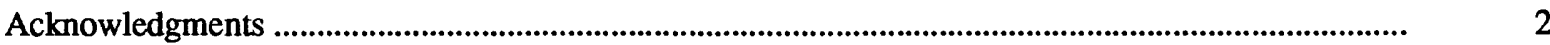

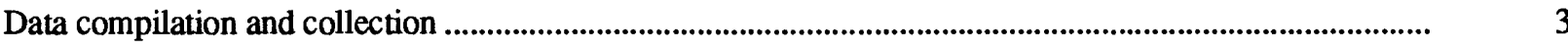

Selected references..................................................................................................................................

\section{ILLUSTRATIONS}

Figure 1. Map showing location of Carroll County and the 16-county area of northern Arkansas................. 1

2. Map showing 7.5-minute topographic quadrangle coverage of Carroll County, Arkansas............ $\quad 4$

3. Diagram showing local well-numbering system ............................................................................

4-7. Maps showing:

4. Locations of wells in Carroll County, Arkansas, for which hydrogeologic data are available ..................................................................................................................

5. Locations of selected springs in Carroll County, Arkansas .....................................................

6. Locations of selected springs in the vicinity of Eureka Springs, Arkansas...............................

7. General areas of sinkholes and internally-drained, closed depressions identified on

7.5-minute topographic quadrangle maps of Carroll County, Arkansas................................

8. Hydrographs showing temporal variation of water levels in selected wells in Carroll

County, Arkansas

\section{TABLES}

Table 1. Description of selected wells for which hydrogeologic data are available,

Carroll County, Arkansas

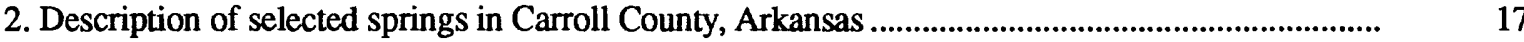

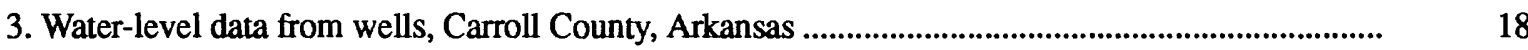

4. Discharge data from springs, Carroll County, Arkansas.............................................................. 25

5. Concentrations of major constituents and properties in water from selected wells and

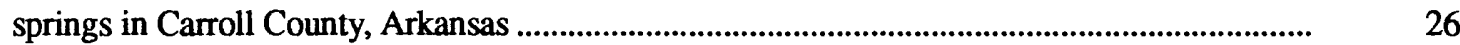

6. Concentrations of trace constituents in water from selected wells and springs in

Carroll County, Arkansas .......................................................................................................................

7. Concentration of nutrients and microorganisms in water from selected wells and springs

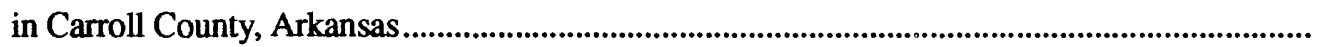

8. Identification of general areas of sinkholes and internally-drained, closed depressions identifiable on 7.5-minute topographic quadrangle maps in Carroll County, Arkansas 


\section{CONVERSION FACTORS AND VERTICAL DATUM}

Multiply

square mile $\left(\mathrm{mi}^{2}\right)$

foot (ft)

gallon (gal)

gallon per minute (gal/min)
By

2.590

0.3048

3.785

0.06309
To obtain

square kilometer

meter

liter

liter per second

Temperature in degrees Celsius $\left({ }^{\circ} \mathrm{C}\right)$ can be converted to degrees Fahrenheit $\left({ }^{\circ} \mathrm{F}\right)$ as follows:

$$
{ }^{\circ} \mathrm{F}=1.8 \times{ }^{\circ} \mathrm{C}+32
$$

Sea level: In this report, "sea level" refers to the National Geodetic Vertical Datum of 1929--a geodetic datum derived from a general adjustment of the first-order level nets of the United States and Canada, formerly called Sea Level Datum of 1929. 


\title{
HYDROGEOLOGIC DATA FOR CARROLL COUNTY, ARKANSAS
}

\author{
By J.V. Brahana, Valarie A. Leidy, John Lindt, and S.A. Hodge
}

\begin{abstract}
This report is a compilation of existing and new hydrogeologic data for Carroll County, Arkansas. Data presented include water levels and water quality from selected wells; discharge and water quality from selected springs; geologic and geophysical data relevant to defining a hydrogeologic framework; and surface aspects of selected karst features. Water-quality data include (1) major constituents and properties, (2) trace constituents, and (3) nutrients, microorganisms, and miscellaneous constituents.
\end{abstract}

\section{INTRODUCTION}

Carroll County, with a total area of $634 \mathrm{mi}^{2}$, is located in north-central Arkansas, bordered by Missouri on the north, Benton County on the west, Newton and Madison Counties on the south, and Boone County on the east (fig. 1). Ground water from wells and springs represents an important resource in Carroll County, providing water for domestic, stock, recreation, and public supplies. Public water supplies currently (1992) using ground water are Davis Water System, Mundell Heights Waterworks, Holiday Island Waterworks, Green Forest Waterworks, Sylvan Shores Water System, Reavis Water System, and Sooter Water System (T.W. Holland, U.S. Geological Survey, written commun., 1992; Will Schell, Schell Drilling Company, oral commun., 1992). In the last several years, several ground-water supplies were replaced by surface water from Beaver Reservoir supplied by the Carroll-Boone Water District (Will Schell, Schell Drilling Company, oral commun., 1992).

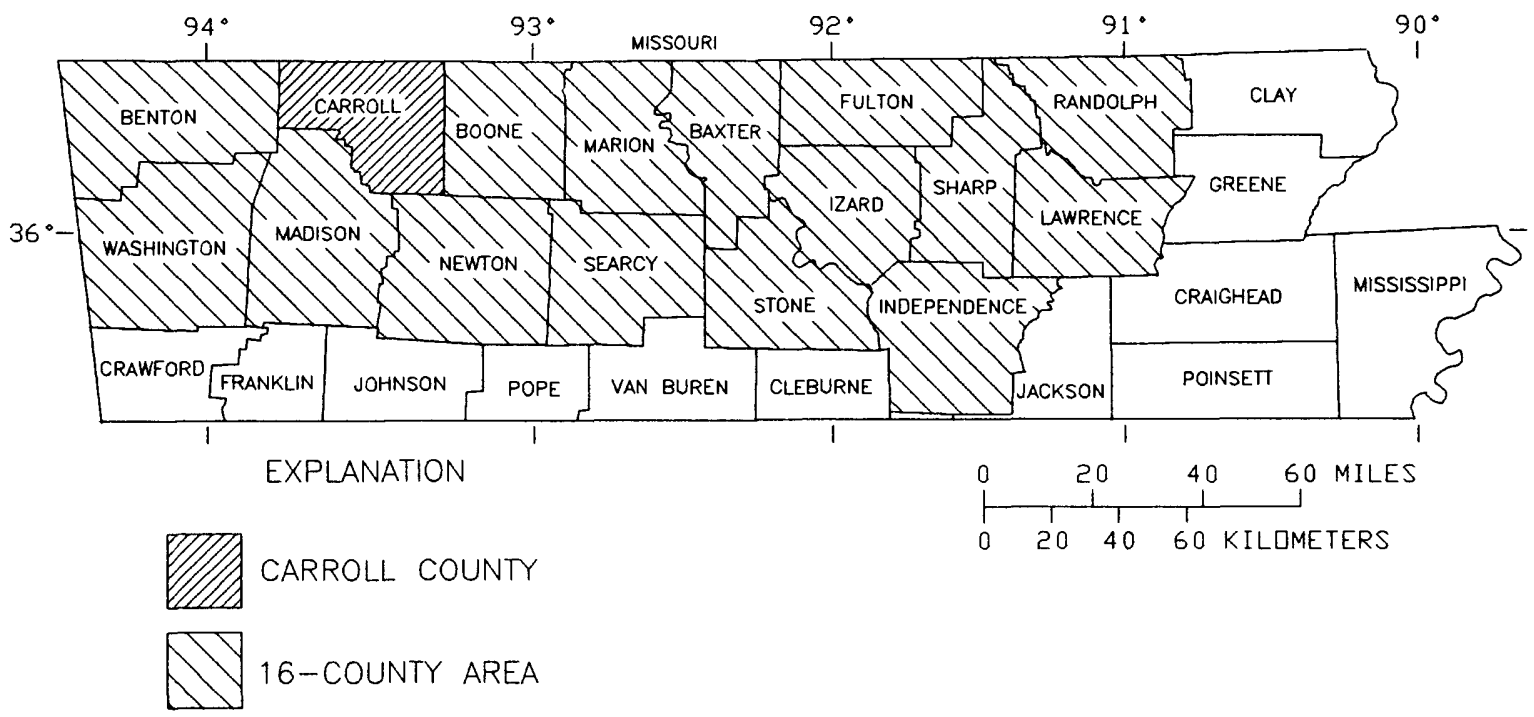

Figure 1.--Location of Carroll County and the 16-county area of northern Arkansas.

Recent population growth in Carroll County has resulted in numerous changes in land use, many of which may affect the ground-water resources. In the last 10 years, population has increased about 15 percent, from 16,203 in 1970 (Fowlkes and others, 1981) to 18,654 in 1990 (Santi, 1990). The economic base of the county is also expanding. Historically, beef cattle production, small industry, and business were the primary agricultural sources of income (Fowlkes and others, 1981). Recently, tourism and poultry production are becoming more important. Chicken and turkey broiler production is expected to increase, which could have a significant effect on ground-water quality. 


\section{Objective and Scope}

The objective of this report is to present a compilation of hydrogeologic data for Carroll County, Arkansas, that can be used to better define and manage the ground-water resources. Prior to this compilation, existing data were scattered through numerous published and unpublished sources, many of which had limited distribution and were difficult to acquire. In anticipation of a needed data base that can be readily accessed and easily used, the Arkansas Soil and Water Conservation Commission and the U.S. Geological Survey entered into a cooperative agreement to compile existing data for a 16-county area of northern Arkansas (fig. 1), and to collect additional data where few data exist. This report is the second in a series of county reports. Hydrogeologic data for Boone County were published in 1991. Carroll County was chosen because it has more existing data than most of the nearby counties and its hydrogeology encompasses most conditions found in the 16-county area. Subsequent reports will present data from the remaining 14 counties.

This report is restricted to basic ground-water data, and no interpretations or explanations are included. The types of data in this report are: information about wells and springs from which data or samples were collected, ground-water levels, spring discharge measurements, water-quality analyses for wells and springs, and surface aspects of selected karst features. In addition, published references with geophysical log and geologic log descriptions necessary for understanding the hydrogeologic framework are included in the report.

The data presented herein have been selected from verifiable sources, including published reports, unpublished records from the files of the U.S. Geological Survey, and new data collected specifically for this study. Hydrogeologic data from sources outside the U.S. Geological Survey generally have not been included in the accompanying tables. However, reference to these original documents, many of which contain data tables, is included in the Previous Investigations and Selected References sections that follow.

\section{Previous Investigations}

Purdue and Miser (1916) described ground water in a cursory manner in their geologic folio of HarrisonEureka Springs. A short list of water-well depths and estimated yields was compiled by the Arkansas Geological Commission, as were major springs (Branner, 1937a; 1937b). Caplan (1957) provided structural interpretation of an area that included Carroll County, thereby refining understanding of the hydrogeologic framework. Lamonds and Stephens (1969) presented hydrogeologic data from northern Arkansas, including Carroll County, in a reconnaissance report of the Ozark Plateau Province; Lamonds (1972) interpreted these data in a hydrologic atlas. MacDonald and others (1975) conducted a ground-water inventory that focused on the deeper aquifers of northern Arkansas, specifically the Roubidoux, Gasconade, and Van Buren Formations. Ogden and others (1979) published a preliminary description of rural use of the aquifers in Boone, Carroll, and Madison Counties based on driller's lithologic logs from water wells.

In addition to the studies mentioned, the U.S. Geological Survey, in cooperation with the Arkansas Geological Commission and other local, State, and Federal agencies, has collected ground-water data from Carroll County that are part of a statewide data base. Some of these data have not been published previously; many have been published in annual basic-data reports for the entire State. All are in computerized data bases of the U.S. Geological Survey (GWSI, WATSTORE), and may be accessed using U.S. Geological Survey retrieval programs.

\section{Acknowledgments}

The authors would like to thank the U.S. Soil Conservation Service, Carroll County Conservation District, for their assistance in this project. Will Schell provided valuable information about ground water and wells in the county, and we gratefully acknowledge the sharing of this extensive information. In addition, the authors are grateful to those property owners throughout the county who allowed sampling and measuring of their wells and springs. 


\section{DATA COMPILATION AND COLLECTION}

As described previously, historic hydrogeologic data were compiled by the U.S. Geological Survey as part of an ongoing program to assess the water resources of Arkansas. These data were collected using standard procedures (Carter and Davidian, 1968; Garber and Koopman, 1968; Keys and MacCary, 1971) and were entered into the data bases of the U.S. Geological Survey.

This historic data base was supplemented by new data collected specifically for this study. Potential datacollection sites were identified by personnel of the U.S. Soil Conservation Service in Carroll County. Each site was field located by U.S. Geological Survey personnel, specific data requirements were assessed, and the site was ranked with respect to its effectiveness in filling data gaps, both areally and stratigraphically. Field verification and data collection at appropriate sites by U.S. Geological Survey personnel completed this data-collection phase.

Descriptive data for the wells in Carroll County have been compiled in table 1 (tables 1 through 8 are located at end of report). As an aid to locating ground-water sites, the locations of 7.5-minute topographic quadrangles in the county are shown in figure 2 . The local well-numbering system, which uses the township-range-section method of locating wells, has been included as an aid to those readers who may need to locate data on maps of varying scales (fig. 3). The local well number has been the most commonly used location identifier in previous studies in Carroll County. This identification method is also applied to the location of springs.

This report uses a site number, a one- to three-digit number whose general location is shown on figure 4; specific locations of wells can be determined from either the local well number or the site ID (table 1), the first 13 digits of which represent the latitude and longitude of the well. Sequential site numbers were assigned arbitrarily at the start of this project to all wells and springs that could potentially be included in this report. Those sites for which data could not be verified were omitted from subsequent tables and maps, and their site numbers were not reassigned, which resulted in gaps in site numbers on tables and figures. Corresponding data for selected springs are compiled in table 2. Locations for springs are shown on figure 5. Springs in the vicinity of Eureka Springs are shown on figure 6.

Water-level data from wells in Carroll County are given in table 3, along with supplemental data listing date of the measurement and the deepest hydrogeologic unit to which the well is open. Discharge data from individual springs are summarized in table 4.

Analyses of ground-water samples were provided by laboratories of the U.S. Geological Survey in Arvada, Colorado; Doraville, Georgia; Ocala, Florida; and Reston, Virginia. Samples were collected, treated, and analyzed using established procedures (Wershaw and others, 1987; Britton and Greeson, 1987; Skougstad and others, 1979; Friedman and Erdmann, 1982; Claassen, 1982). Temperature, $\mathrm{pH}$, and alkalinity (table 5) were determined in the field using established field procedures (Stevens and others, 1975; Wood, 1976).

Ion chromatography was used to determine the major anions. Trace-constituent concentrations (table 6) were determined by using inductively coupled plasma atomic emission spectroscopy (ICP). Nutrients, microorganisms, and miscellaneous constituents in water from selected wells and springs are summarized in table 7.

Most of the analytical values are in standard reporting units such as milligrams per liter or micrograms per liter. These data are stored in the U.S. Geological Survey's WATSTORE data base, and may be accessed using Geological Survey retrieval programs.

Well locations for tables 1, 3, 5, 6, and 7 are shown in figure 4, and spring locations for tables 2, 4, 5, 6, and 7 are shown on figures 5 and 6. Selected karst features in Carroll County are summarized in table 8, and their locations are shown on figure 7.

Temporal variation of water levels in selected wells in Carroll County are shown in figure 8. Periodic measurements of the depth to water in these wells were obtained from December 1990 through August 1992. 


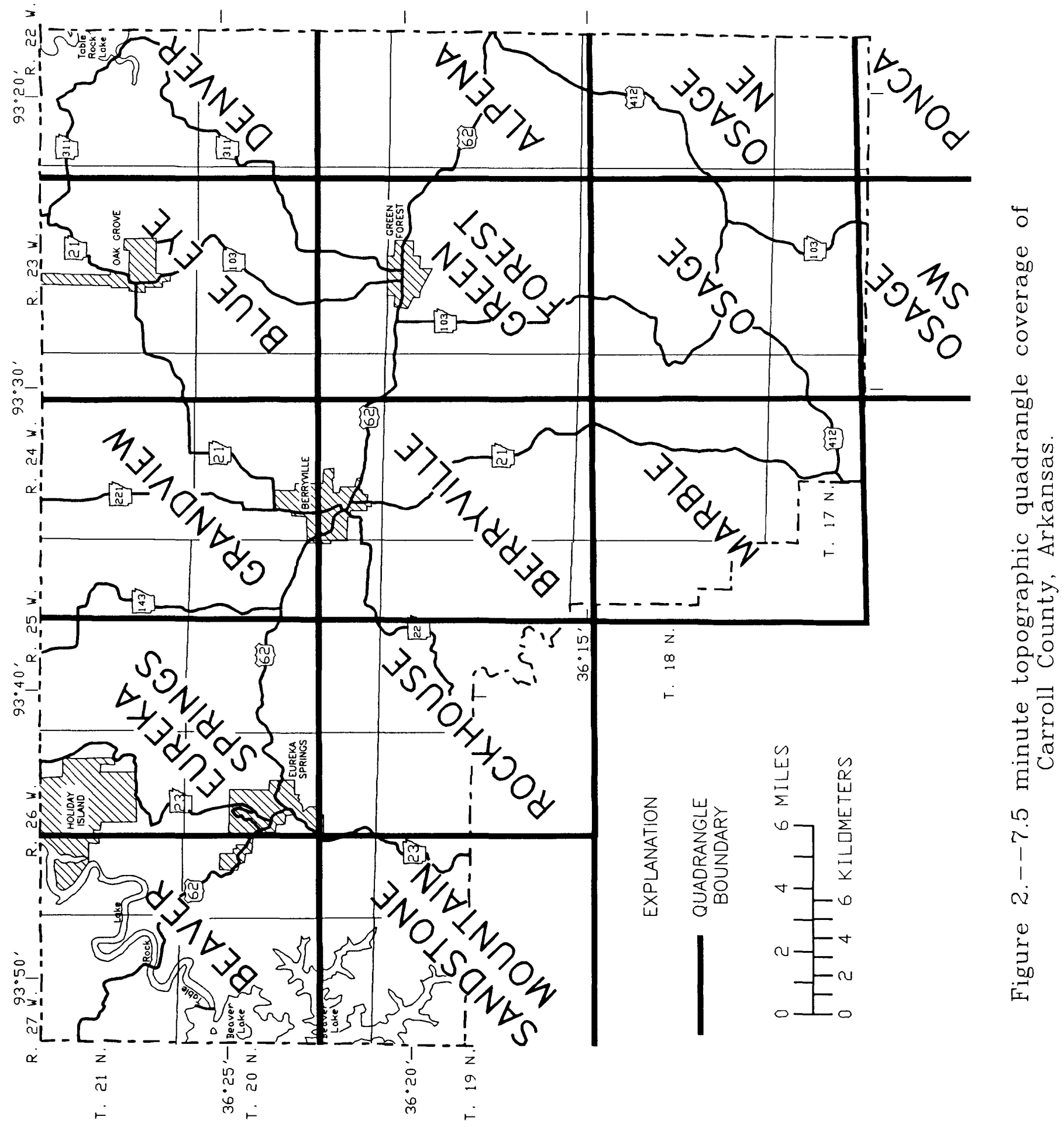




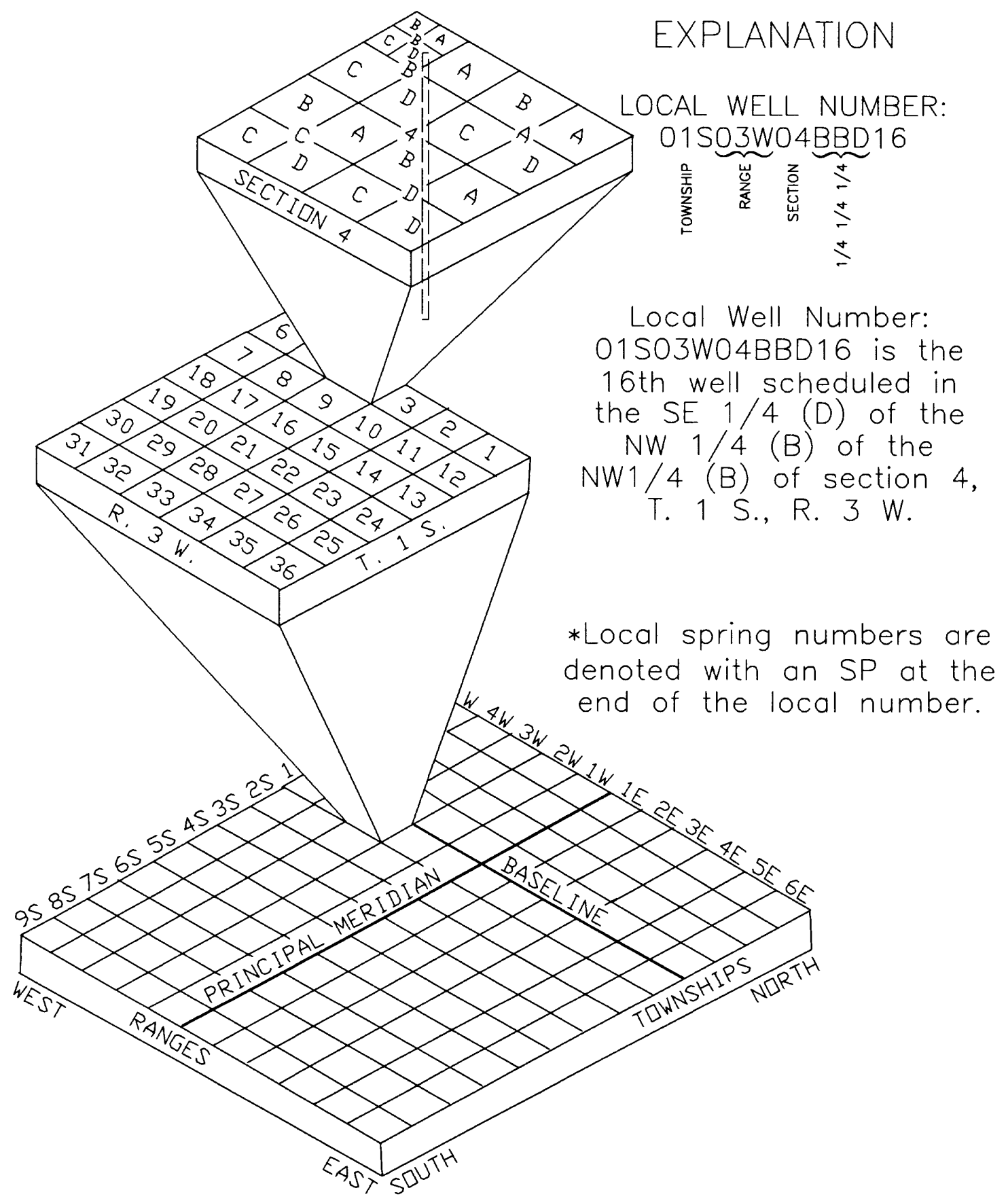

Figure 3.--Local well-numbering system. 


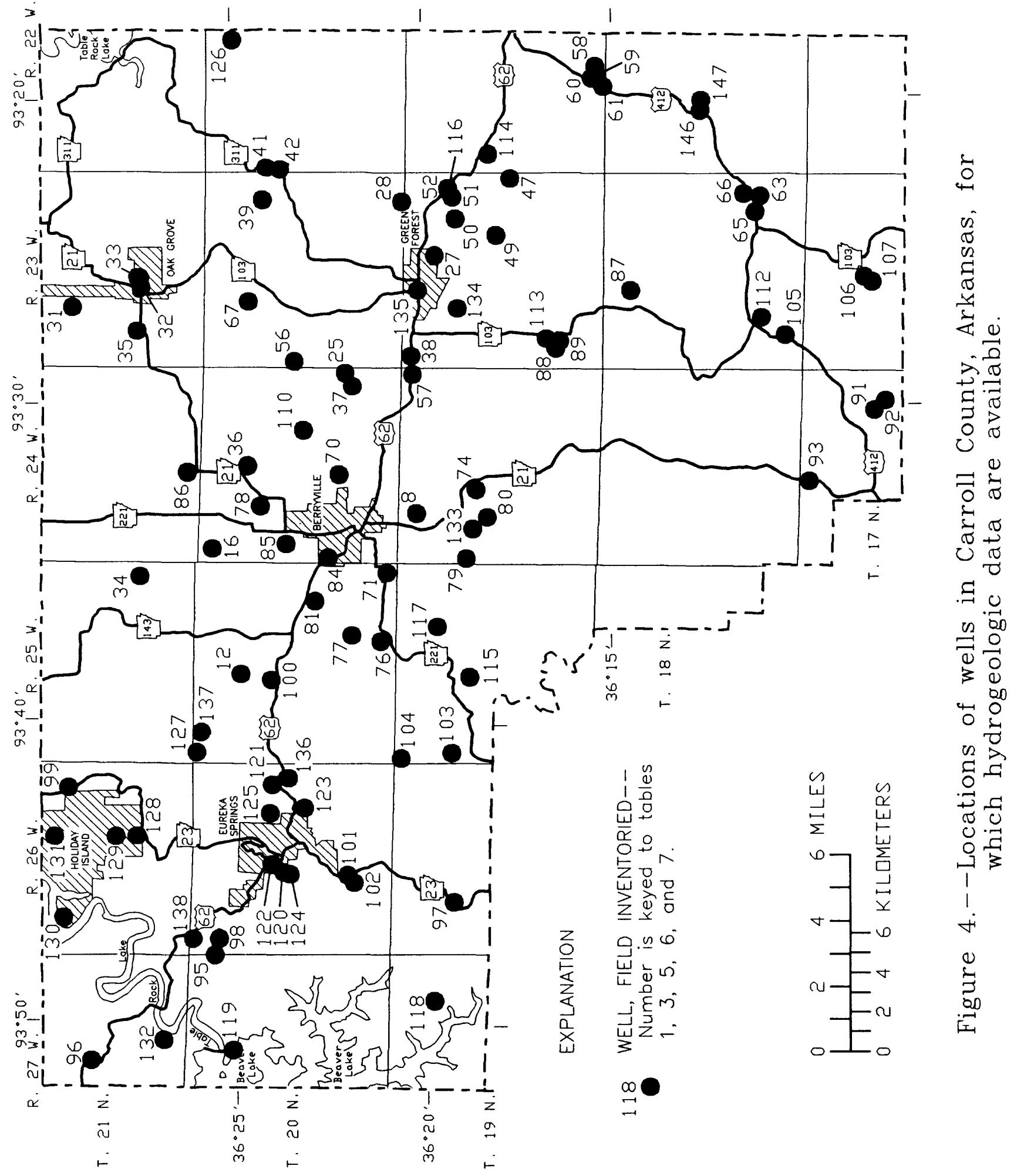




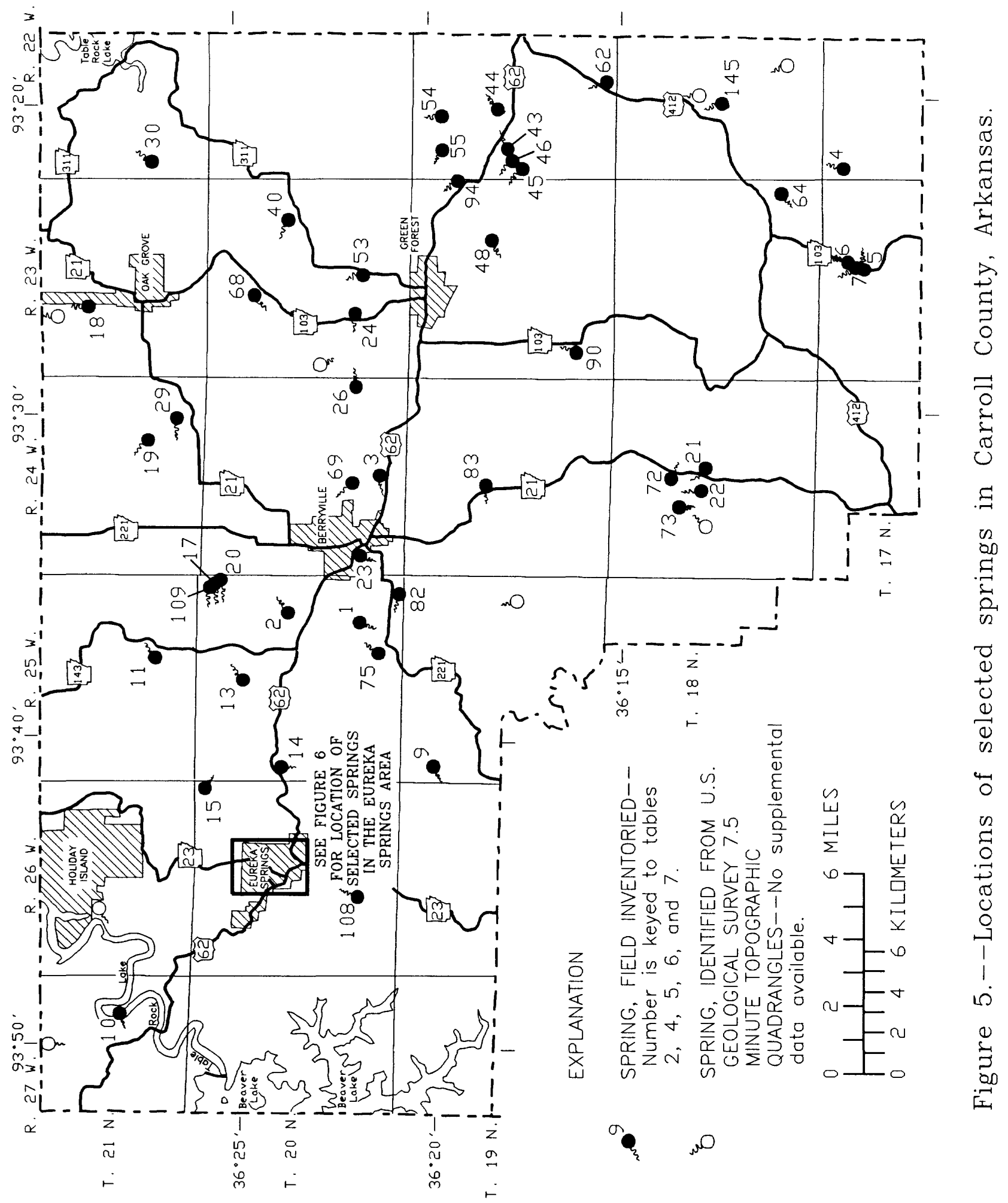




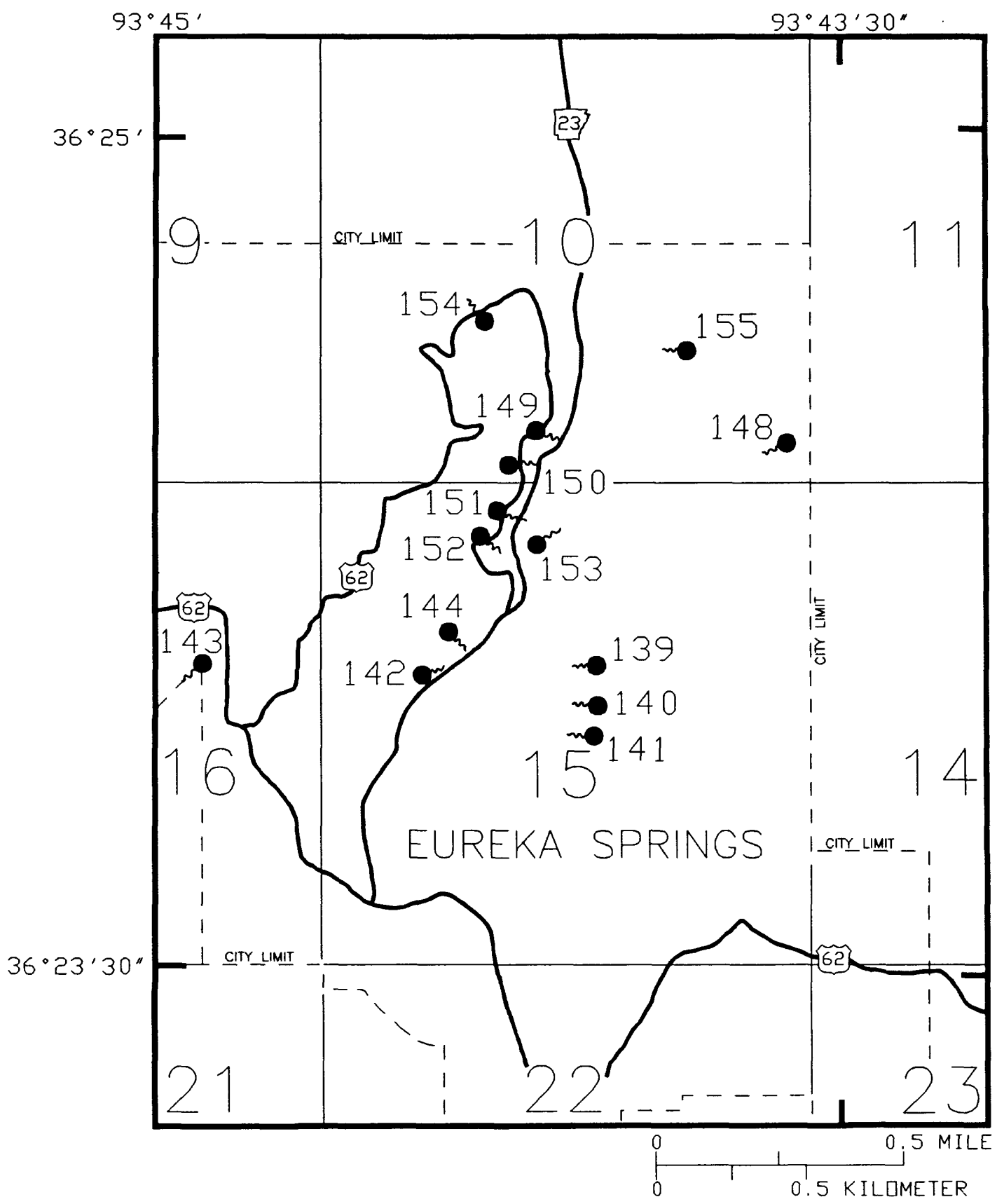

EXPLANATIDN

SPRING, FIELD INVENTIRIED--
Number is keyed to tables $2,4,5,6$, and 7 .

15 SECTIIN NUMBER--Section in T. $20 \mathrm{~N}$. and R. $26 \mathrm{~W}$.

Figure 6.--Locations of selected springs in the vicinity of Eureka Springs, Arkansas. 


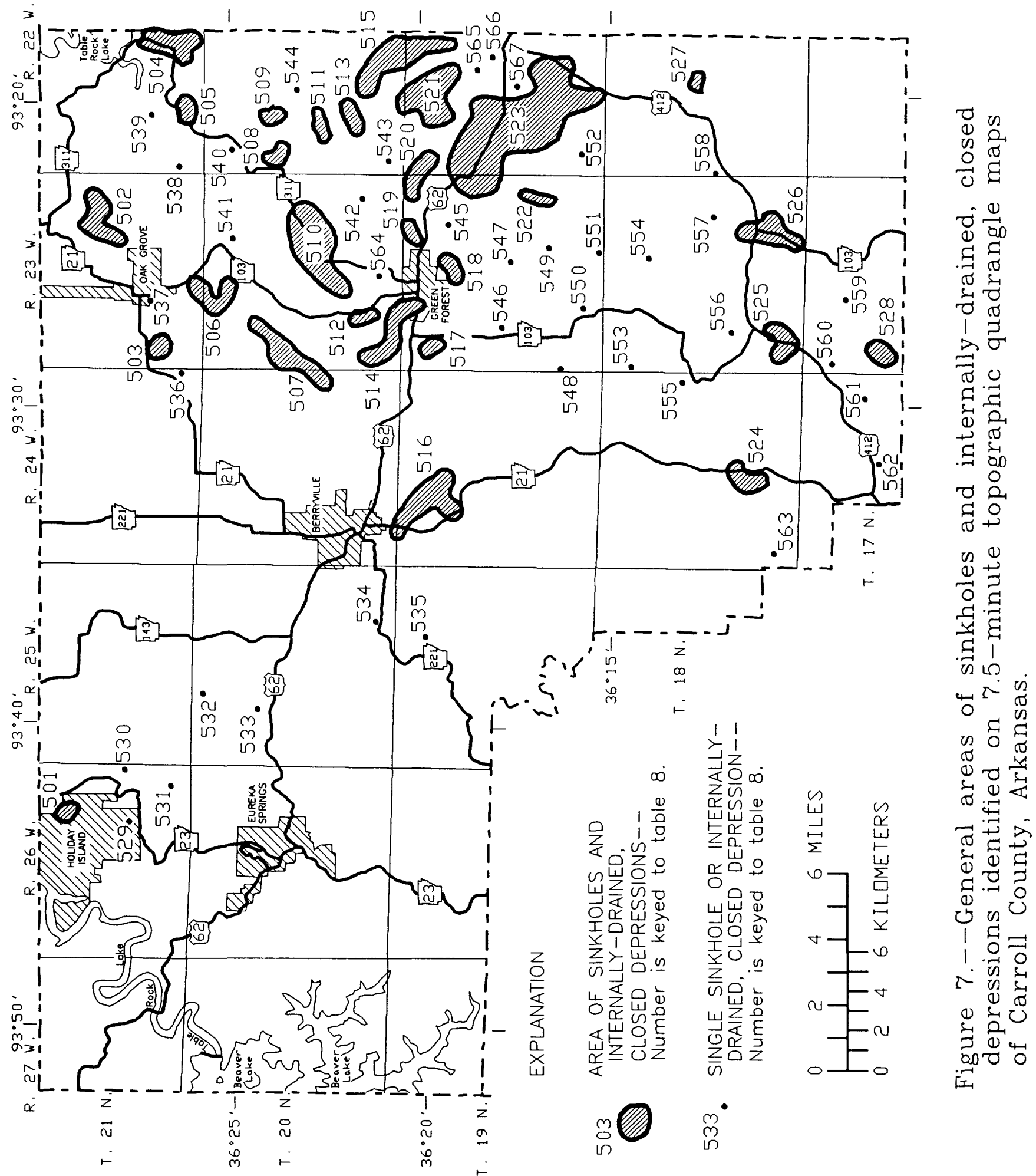




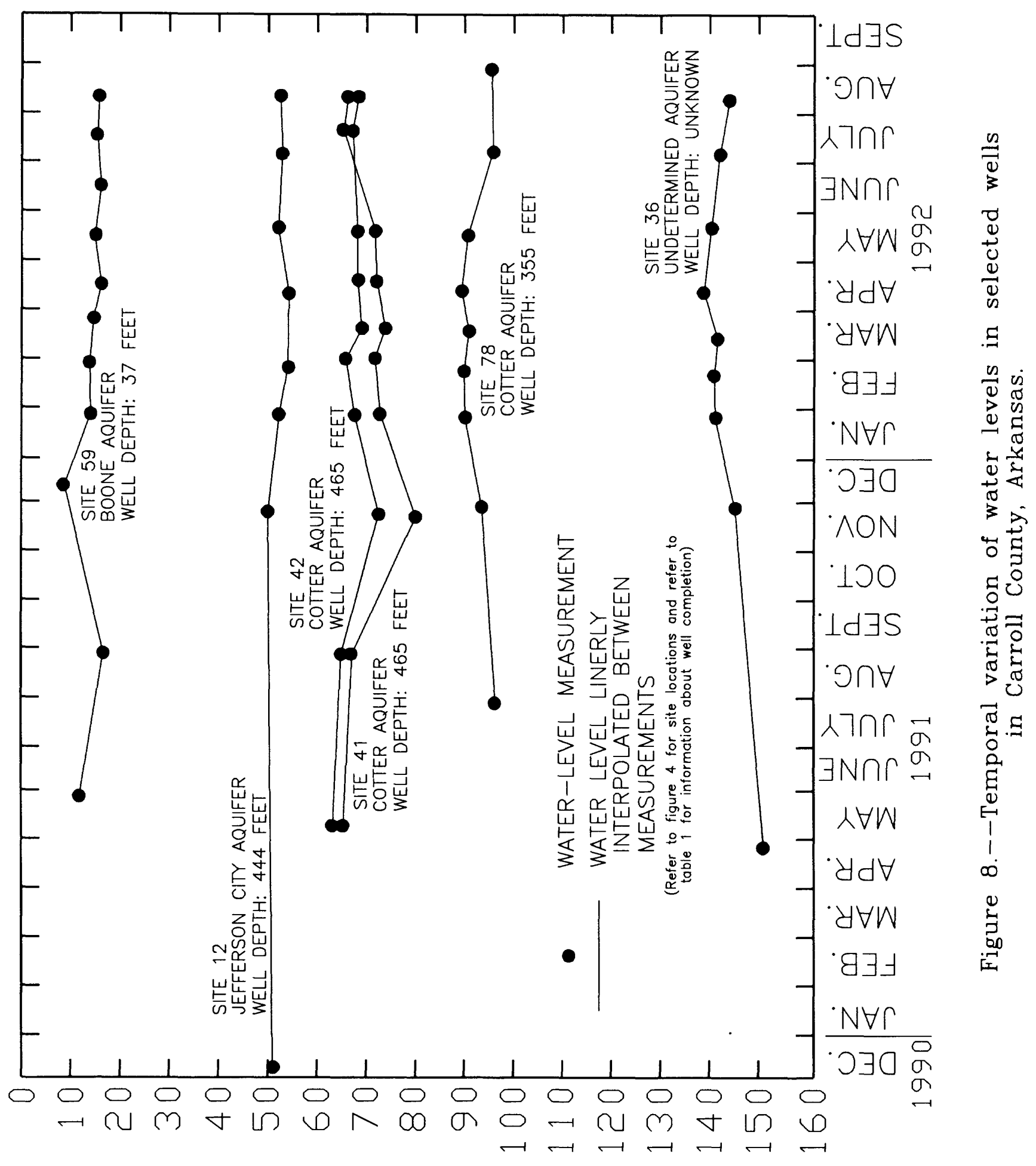

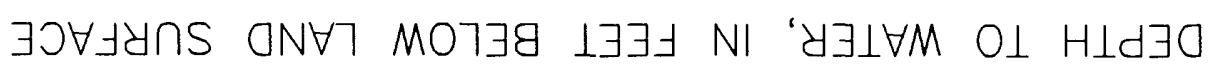




\section{SELECTED REFERENCES}

Brahana, J.V., Gonthier, G.J., and Remsing, L.M., 1991, Hydrogeologic data for Boone County, Arkansas: U.S. Geological Survey Open-File Report 91-518, 36 p.

Branner, G.C., 1937a, List of Arkansas water wells: Arkansas Geological and Conservation Commission Information Circular 11, $142 \mathrm{p}$.

---- 1937b, Data on springs in Arkansas: Arkansas Geological and Conservation Commission unnumbered series, $127 \mathrm{p}$.

Britton, L.J., and Greeson, P.E., eds., 1987, Methods for collection and analysis of aquatic biological and microbiological samples: U.S. Geological Survey Techniques for Water-Resources Investigations, Book 5, Chapter A4, $363 \mathrm{p}$.

Bush, W.V., Haley, B.R., Stone, C.G., Holbrook, D.F., and McFarland, J.D. III, 1977, A guidebook to the geology of the Arkansas Paleozoic area (Ozark Mountains, Arkansas Valley, and Ouachita Mountains): Arkansas Geological Commission Guidebook 77-1, 79 p.

Caplan, W.M., 1957, Subsurface geology of northwestern Arkansas: Arkansas Geological and Conservation Commission Information Circular 19, 14 p.

-...- 1960, Subsurface geology of pre-Everton rocks in northern Arkansas: Arkansas Geological and Conservation Commission Information Circular 21, 17 p.

Carter, R.W., and Davidian, Jacob, 1968, General procedure for gaging streams: U.S. Geological Survey Techniques of Water-Resources Investigations, Book 3, Chapter A6, 13 p.

Chazen, B., and Erickson, M.S., 1988, Spectrographic analyses of insoluble residue samples, Harrison $1^{\circ} \times 2^{\circ}$ quadrangle, Missouri and Arkansas--Drill holes nos. 64, 65, 66, 67: U.S. Geological Survey Open-File Report $88-529,37 \mathrm{p}$.

Claassen, H.C., 1982, Guidelines and techniques for obtaining water samples that accurately represent the chemistry of an aquifer: U.S. Geological Survey Open-File Report 82-1024, 49 p.

Croneis, Carey, 1930, Geology of the Arkansas Paleozoic area: Arkansas Geological Survey annual report, v. 4, $457 \mathrm{p}$.

Dillard T., 1978, The geohydrology and water quality of the Upper Buffalo River Basin, Newton County, Arkansas: University of Arkansas, Fayetteville, Arkansas, unpublished M.S., thesis, 99 p.

Fowlkes, D.H., Harper, M.D., and McCright, R.T., 1981, Soil survey of Carroll County, Arkansas: U.S. Department of Agriculture, $119 \mathrm{p}$.

Freiwald, D.A., 1987, Streamflow gain and loss of selected streams in northern Arkansas: U.S. Geological Survey Water-Resources Investigations Report 86-4185, 4 sheets.

Friedman, L.C., and Erdmann, D.E., 1982, Quality assurance practices for the chemical and biological analyses of water and fluvial sediments: U.S. Geological Survey Techniques of Water-Resources Investigations, Book 5, Chapter A6, $181 \mathrm{p}$.

Garber, M.S., and Koopman, F.C., 1968, Methods of measuring water levels in deep wells: U.S. Geological Survey Techniques of Water-Resources Investigations, Book 8, Chapter A1, 23 p.

Haley, B.R., 1976, Geologic map of Arkansas: U.S. Geological Survey, scale 1:500,000.

Harvey, E.J., 1980, Ground water in the Springfield-Salem Plateaus of southern Missouri and northern Arkansas: U.S. Geological Survey Water-Resources Investigations Report 80-101, 66 p.

Hedman, E.R., Skelton, John, and Freiwald, D.A., 1986, Flow characteristics for selected springs and streams in the Ozark subregion, Arkansas, Kansas, Missouri, and Oklahoma: U.S. Geological Survey Hydrologic Investigations Atlas HA-688, 4 sheets. 
Imes, J.L., 1988, Geohydrology and hydrochemistry of the Ozark Plateaus aquifer system: American Water Resources Association, Regional Aquifer Systems of the United States--Aquifers of the midwestern area, p. 165-178.

Keys, W.S., and MacCary, L.M., 1971, Application of borehole geophysics to water-resources investigations: U.S. Geological Survey Techniques of Water-Resources Investigations, Book 2, Chapter E1, $126 \mathrm{p}$.

Kilpatrick, J.M., and Ludwig, A.H., 1990, Ground-water resources of the White River Basin in Arkansas: U.S. Geological Survey Open-File Report 88-724, 48 p.

Lamonds, A.G., 1972, Water-resources reconnaissance of the Ozark Plateaus Province, northern Arkansas: U.S. Geological Survey Hydrologic Investigations Atlas HA-383, 2 sheets.

Lamonds, A.G., and Stephens, J.W., 1969, Water-resources data for the Ozark Plateaus Province, Arkansas: U.S. Geological Survey open-file report, $18 \mathrm{p}$.

Leidy, V.A., and Morris, E.E., 1990, Hydrogeology and quality of ground water in the Boone Formation and Cotter Dolomite in karst terrain of northwestern Boone County, Arkansas: U.S. Geological Survey Water-Resources Investigations Report 90-4066, 57 p.

MacDonald, Harold, Zachry, D.L., and Jeffus, Hugh, 1975, Northern Arkansas groundwater inventory: Arkansas Water Resources Research Center, Miscellaneous Bulletin 26, Fayetteville, Arkansas, 186 p.

McCafferty, D.E., Cordell, L., and Bracken, R.E., 1989, Geophysical maps and interpretation of basement terrance in the Harrison $1^{\circ} \times 2^{\circ}$ quadrangle, Missouri and Arkansas: U.S. Geological Survey Miscellaneous Field Studies Map, scale 1:250,000.

McFarland, J.D., III, Bush, W.V., Wise, Orville, and Holbrook, Drew, 1979, A guidebook to the OrdovicianMississippian rocks of north-central Arkansas: Arkansas Geological Commission, GB-79-1, 29 p.

Melton, R.W., 1976, The regional geohydrology of the Roubidoux and Gasconade Formations, Arkansas and Missouri: University of Arkansas, Fayetteville, Arkansas, unpublished M.S. thesis, 160 p.

Ogden, A.E., Taylor, N.L., and Thompson, S., 1979, A preliminary investigation of the rural-use aquifers of Boone, Carroll, and Madison Counties, Arkansas: Proceedings of the Arkansas Academy of Science, p. 58-60.

Purdue, A.H., and Miser, H.D., 1916, Description of Eureka Springs and Harrison quadrangles (Arkansas-Missouri): U.S. Geological Survey Geologic Atlas, Folio 202, 21 p.

Santi, L.L., 1990, An analysis of preliminary 1990 census counts for the State of Arkansas: Division of Demographic Research, Little Rock, 45 p.

Sheldon, M.G., 1954, Sample descriptions and correlations for selected wells in northern Arkansas: Arkansas Geological Commission Information Circular No. 17, p. 222.

Skougstad, M.W., Fishman, M.J., Friedman, L.C., Erdmann, D.E., and Duncan, S.S., 1979, Methods for determination of inorganic substances in water and fluvial sediment: U.S. Geological Survey Techniques of Water-Resources Investigations, Book 5, Chapter A1, $626 \mathrm{p}$.

Snyder, R.W., 1976, The stratigraphy of the Roubidoux and Gasconade Formations of northern Arkansas: University of Arkansas, Fayetteville, Arkansas: unpublished M.S. thesis, 90 p.

Steele, K.F., and Adamski, J.C., 1987, Landuse effects of ground-water quality in carbonate terrain: Arkansas Water Resources Research Center Publication No. 129, 71 p.

Stevens, H.H., Jr., Ficke, J.F., and Smoot, G.F., 1975, Water temperature--influential factors, field measurement, and data presentation: U.S. Geological Survey Techniques of Water-Resources Investigations, Book 1, Chapter D1, $65 \mathrm{p}$.

Tisdale, T.J., 1975, The geography of Carroll County, Arkansas: University of Arkansas, Fayetteville, Arkansas: unpublished M.S. thesis, $78 \mathrm{p}$.

U.S. Environmental Protection Agency, 1986, Amendment to national oil and hazardous substances contingency plan; national priorities list, final rule, and proposed rule: Federal Register, v. 51, no. III, June 10, 1986, p. 21053-21112. 
Webb, J.H., 1961, The geology of Green Forest Township, Carroll County, Arkansas: University of Arkansas, Fayetteville, Arkansas, unpublished M.S. thesis, 59 p.

Wershaw, R.L., Fishman, M.J., Grabbe, R.R., and Lowe, L.E., 1987, Methods for the determination of organic substances in water and fluvial sediments: U.S. Geological Survey Techniques of Water-Resources Investigations, Book 5, Chapter A3, 80 p.

Wood, W.W., 1976, Guidelines for collection and field analysis of ground-water samples for selected unstable constituents: U.S. Geological Survey Techniques of Water-Resources Investigations, Book 1, Chapter D2, 4 p. 


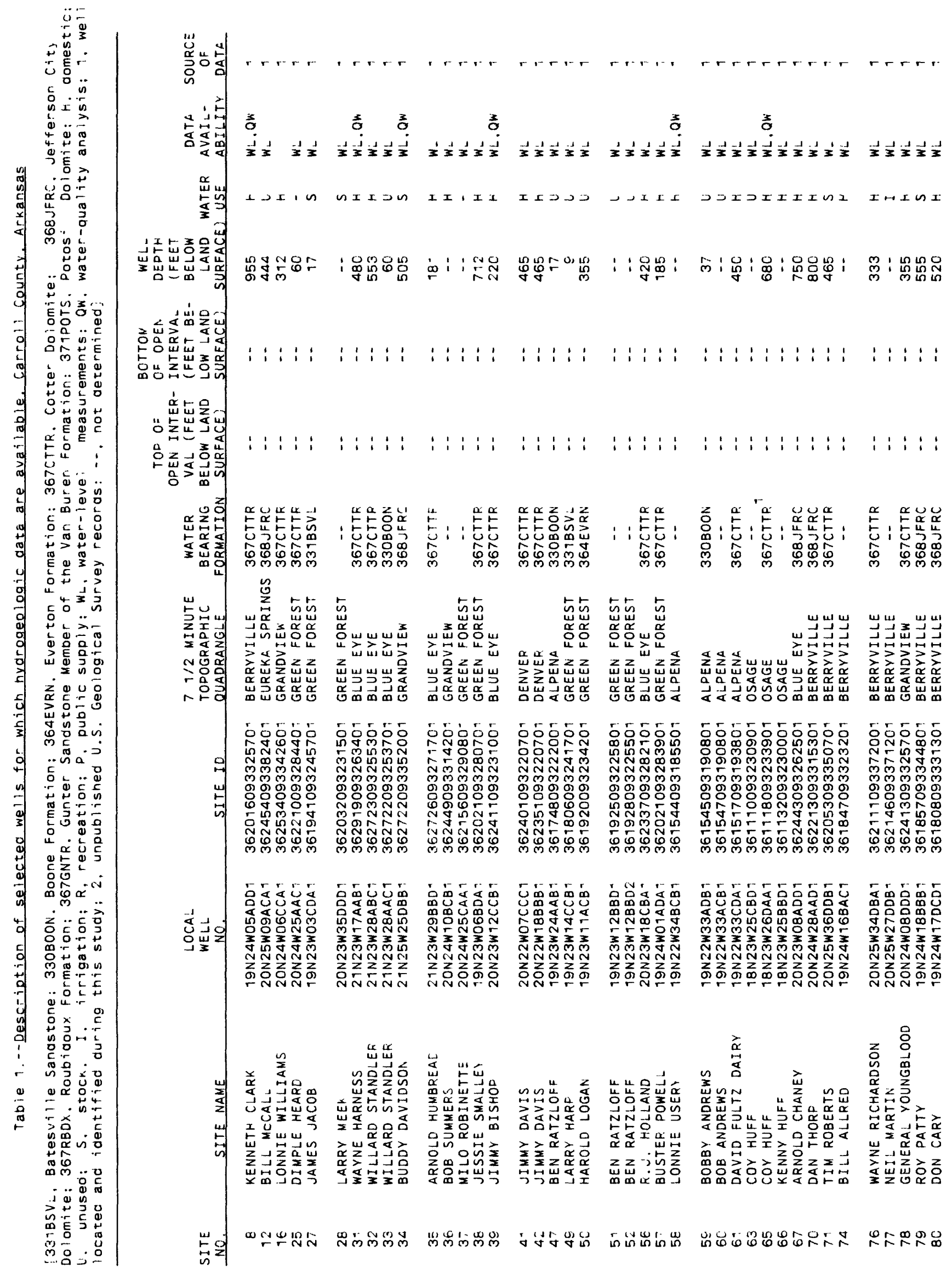




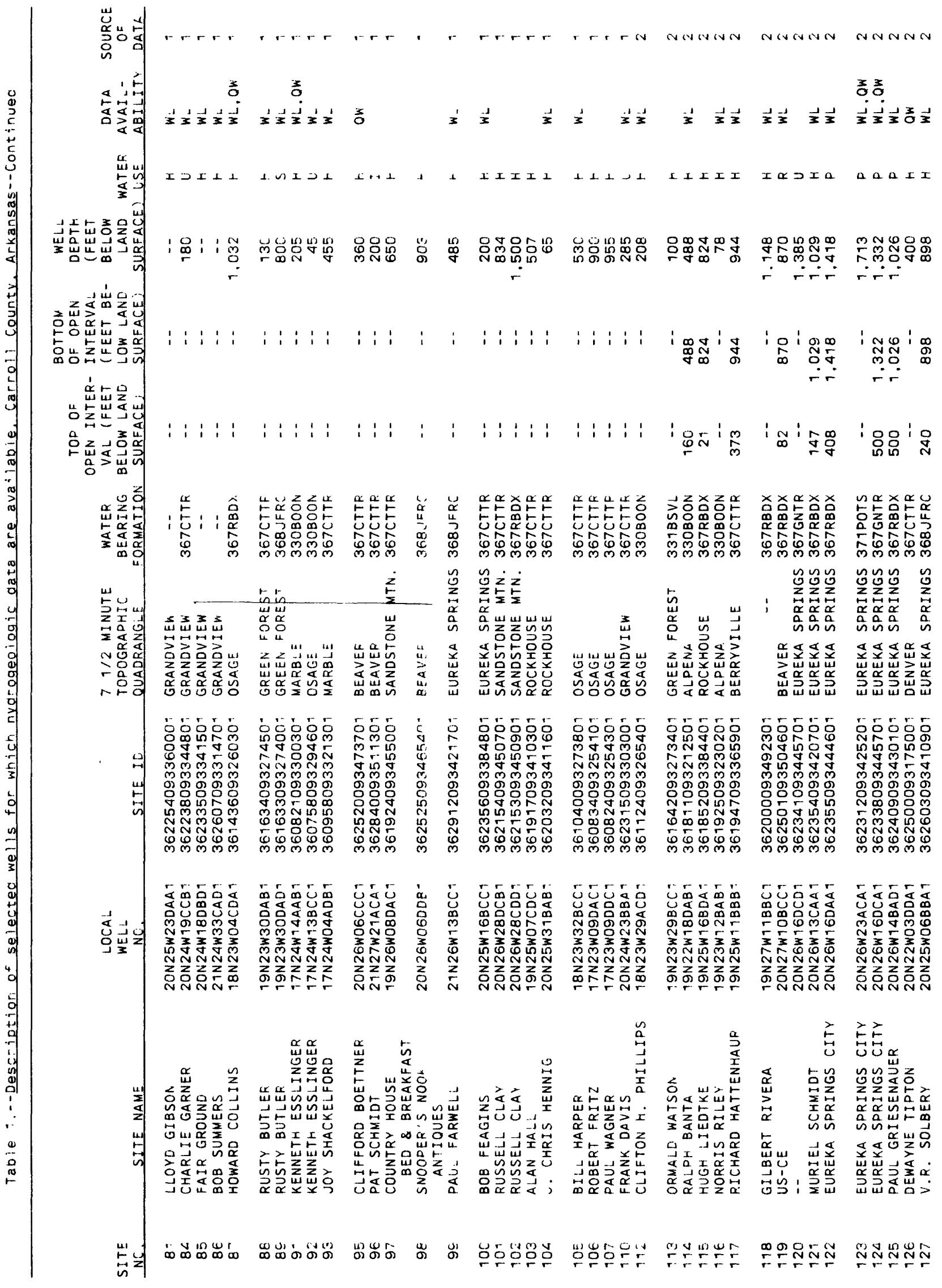




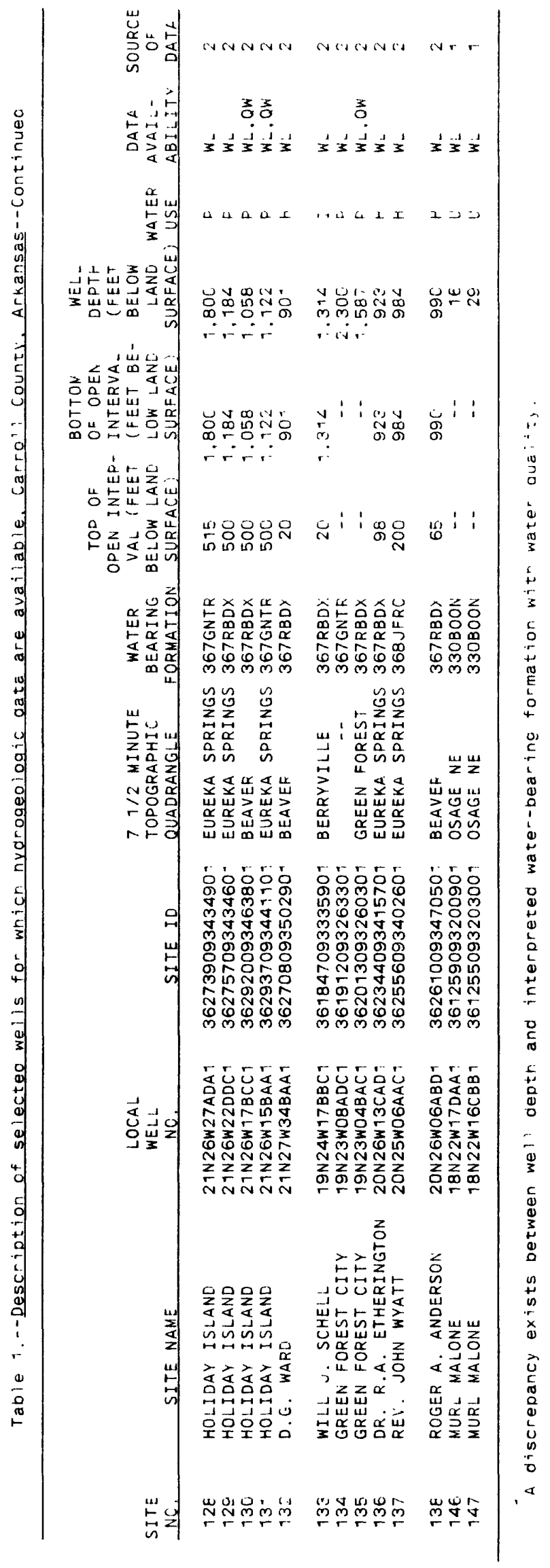


laule 2. - Description ot selected springs in Carroll county. Arkansás

[3318SVL, Hatesville Sandstone; 330800N, Buwne formation; 36/CITR, Cotter Dolomite; P, public supply; $S$, stock; U, unused; R, recreation; H, domeslic; WD, discharge measurement; QW, water-quality analyses; 1, spring localed and identified during this study; 2, Lamonds (1972); --, not determined]

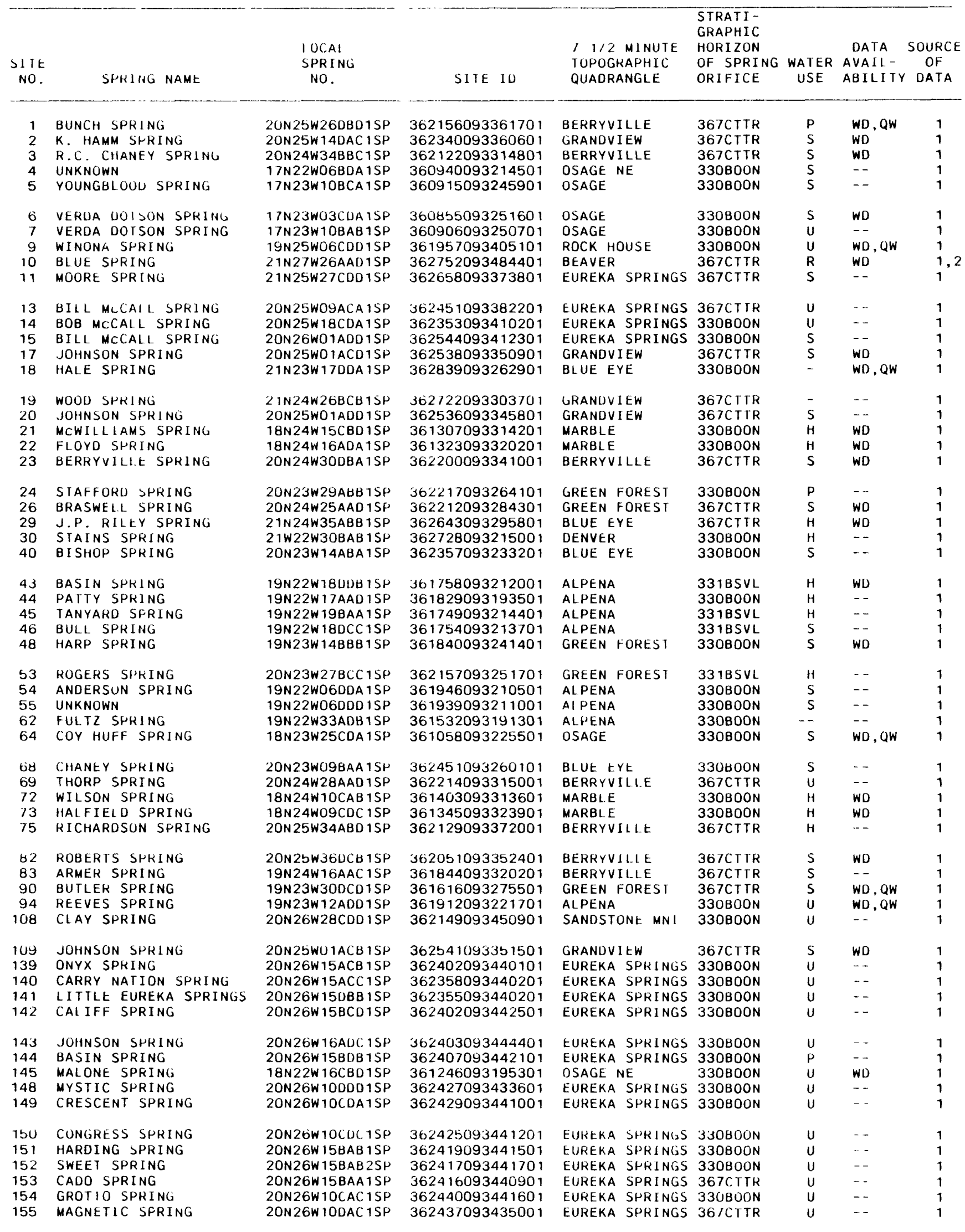



371 POTS, Potosi Dolomite; --, not identified]

\begin{tabular}{|c|c|c|c|c|c|c|}
\hline $\begin{array}{l}\text { SITE } \\
\text { NO. }\end{array}$ & SITE NAME & $\begin{array}{l}\text { AL I I TUDE } \\
\text { OF LAND } \\
\text { SURFACE } \\
\text { (FEET ABOVE } \\
\text { SEA LEVEL) }\end{array}$ & $\begin{array}{c}\text { DEPTH } \\
\text { TO } \\
\text { WATER } \\
\text { (FEET BELOW } \\
\text { LAND SUREACE) }\end{array}$ & $\begin{array}{c}\text { ALTITUDE } \\
\text { OF WATER } \\
\text { IEVEL } \\
\text { (FEET ABOVE } \\
\text { SEA LEVEL) }\end{array}$ & $\begin{array}{l}\text { DATE } \\
\text { WATER LEVEL } \\
\text { MEASURED }\end{array}$ & $\begin{array}{l}\text { WATER- } \\
\text { BEARING } \\
\text { FORMA- } \\
\text { TION }\end{array}$ \\
\hline \multirow[t]{2}{*}{8} & KENNETH CLARK & 1,155 & $\begin{array}{r}10.53 \\
30.75 \\
9.59 \\
11.40 \\
7.10\end{array}$ & $\begin{array}{l}1,144.47 \\
1.124 .25 \\
1: 145.41 \\
1,143.60 \\
1.147 .90\end{array}$ & $\begin{array}{l}11-14-90 \\
08-23-91 \\
09-26-91 \\
11-26-91 \\
01-21-92\end{array}$ & 367 CTTR \\
\hline & & & $\begin{array}{r}7 \cdot 15 \\
7 \cdot 50 \\
8 \cdot 00 \\
15 \cdot 85 \\
24.50\end{array}$ & $\begin{array}{l}1.147 .85 \\
1: 147.50 \\
1,147.00 \\
1: 139.15 \\
1.130 .50\end{array}$ & $\begin{array}{l}02-19-92 \\
03-26-92 \\
04-16-92 \\
05-21-92 \\
07-07-92\end{array}$ & \\
\hline 12 & BII 1 MCCALL. & 1.060 & $\begin{array}{l}16.25 \\
51.10 \\
50.00 \\
51.90 \\
54.10\end{array}$ & $\begin{array}{l}1,138.75 \\
1,008.90 \\
1,010.00 \\
1,008.10 \\
1,005.90\end{array}$ & $\begin{array}{l}08-13-92 \\
12-06-90 \\
11-21-91 \\
01-20-92 \\
02-19-92\end{array}$ & $368 \mathrm{JFRC}$ \\
\hline 25 & DIMHLE HEARD & 1,280 & $\begin{array}{l}53.40 \\
52.00 \\
51.80 \\
52.10 \\
20.34\end{array}$ & $\begin{array}{l}1.006 .60 \\
1.008 .00 \\
1.008 .20 \\
1,007.90 \\
1.259 .66\end{array}$ & $\begin{array}{l}04-16-92 \\
05-26-92 \\
07-07-92 \\
08-13-92 \\
03-12-91\end{array}$ & 367CTTR \\
\hline 28 & $\begin{array}{l}\text { JAMES JACOB } \\
\text { L.ARRY MEEK }\end{array}$ & 1.340 & $\begin{array}{r}6.60 \\
4.00 \\
3.80 \\
3.90 \\
293.90\end{array}$ & $\begin{array}{l}1,333.40 \\
1,336.00 \\
1,336.20 \\
1,336.10 \\
1,046.10\end{array}$ & $\begin{array}{l}03-21-91 \\
12-10-91 \\
01-20-92 \\
02-27-92 \\
03-21-91\end{array}$ & $\begin{array}{c}33 \text { IBSVL } \\
--\end{array}$ \\
\hline \multirow[t]{2}{*}{31} & WAVINE HARNESS & 1.260 & $\begin{array}{l}137.80 \\
144.00 \\
143.08 \\
142.50 \\
142.50\end{array}$ & $\begin{array}{l}1.122 .20 \\
1: 116.00 \\
1.1116 .92 \\
1.117 .50 \\
1.117 .50\end{array}$ & $\begin{array}{l}04-16-91 \\
08-21-91 \\
09-24-91 \\
12-05-91 \\
01-20-92\end{array}$ & $367 C T T R$ \\
\hline & & & $\begin{array}{l}135.90 \\
136.60 \\
135.16 \\
136.50 \\
138.40\end{array}$ & $\begin{array}{l}1.124 .10 \\
1: 123.40 \\
1,124.84 \\
1,123.50 \\
1.121 .60\end{array}$ & $\begin{array}{l}02-18-92 \\
03-17-92 \\
04-09-92 \\
05-14-92 \\
07-23-92\end{array}$ & \\
\hline 32 & WILLARD STANDLER & 1.340 & $\begin{array}{r}137.80 \\
62.45 \\
64.55\end{array}$ & $\begin{array}{l}1.122 .20 \\
1.277 .55 \\
1.275 .45\end{array}$ & $\begin{array}{l}08-11-92 \\
04-16-91 \\
08-21-91\end{array}$ & $367 C T T R$ \\
\hline 33 & WILLARD STANDLER & 1.3111 & $\begin{array}{l}31 \cdot 20 \\
34.37\end{array}$ & $\begin{array}{l}1.278 .80 \\
1.275 .63\end{array}$ & $\begin{array}{l}04-16-9 i \\
08-21-9 i\end{array}$ & $330 B O O N$ \\
\hline \multirow[t]{2}{*}{34} & BUDISY DAVIDSON & 1.0011 & $\begin{array}{r}80.05 \\
99.97 \\
105.46 \\
81.25 \\
86.25\end{array}$ & $\begin{array}{l}979.95 \\
960.03 \\
954.54 \\
978.75 \\
973.75\end{array}$ & $\begin{array}{l}04-23-91 \\
08-21-91 \\
09-24-91 \\
12-05-91 \\
01-20-92\end{array}$ & $368 \mathrm{JFRC}$ \\
\hline & & & $\begin{array}{l}87.10 \\
88.15 \\
81.70 \\
89.60 \\
97.75\end{array}$ & $\begin{array}{l}972.90 \\
971.85 \\
978.30 \\
970.40 \\
962.25\end{array}$ & $\begin{array}{l}02-18-92 \\
03-17-92 \\
04-09-92 \\
05-14-92 \\
07-07-92\end{array}$ & \\
\hline \multirow[t]{2}{*}{$\begin{array}{l}35 \\
36\end{array}$} & $\begin{array}{l}\text { ARNUL.D HUMBREAD } \\
\text { BOE SUMMERS }\end{array}$ & $\begin{array}{l}1,311 \\
1,305\end{array}$ & $\begin{array}{r}94.30 \\
120.00 \\
152.45 \\
146.45 \\
142.50\end{array}$ & $\begin{array}{r}965.70 \\
1.190 .00 \\
1.152 .55 \\
1.158 .55 \\
1.162 .50\end{array}$ & $\begin{array}{l}06-11-92 \\
04-23-91 \\
04-23-91 \\
11-26-91 \\
01-20-92\end{array}$ & $\begin{array}{c}367 C T T R \\
-\end{array}$ \\
\hline & & & $\begin{array}{l}142.00 \\
142.10 \\
138.60 \\
140.20 \\
142.30\end{array}$ & $\begin{array}{l}1,163.00 \\
1,162.90 \\
1,166.40 \\
1,164.80 \\
1,162.70\end{array}$ & $\begin{array}{l}02-18-92 \\
03-17-92 \\
04-09-92 \\
05-21-92 \\
07-07-92\end{array}$ & \\
\hline
\end{tabular}


Table 3. - water level data from wel 1s. Carroll County Arkarisas- Continued

\begin{tabular}{|c|c|c|c|c|c|c|}
\hline $\begin{array}{l}\text { SITE } \\
\text { NU. }\end{array}$ & SITE NAME & $\begin{array}{l}\text { AL TI TUDE } \\
\text { OF LAND } \\
\text { SUKFACE } \\
\text { (FEET ABOVE } \\
\text { SEA IEVEL) }\end{array}$ & $\begin{array}{c}\text { DEPTH } \\
\text { TO } \\
\text { WATER } \\
\text { (FEET BEL OW } \\
\text { LAND SURFACE) }\end{array}$ & $\begin{array}{c}\text { AI I I TUDE } \\
\text { OF WATER } \\
\text { LEVEL } \\
\text { (FEET ABOVE } \\
\text { SEA I EVEL) }\end{array}$ & $\begin{array}{l}\text { DATE } \\
\text { WATER L.EVEL. } \\
\text { MEASURED }\end{array}$ & $\begin{array}{l}\text { WATER- } \\
\text { BEARING } \\
\text { FORMA- } \\
\text { TION }\end{array}$ \\
\hline \multirow[t]{2}{*}{$\begin{array}{l}31 \\
38\end{array}$} & $\begin{array}{l}\text { MIIU ROBINETIE } \\
\text { JESSIE SMALIEY }\end{array}$ & $\begin{array}{l}1,345 \\
1,380\end{array}$ & $\begin{array}{r}143.80 \\
63.80 \\
62.40 \\
61.00 \\
37.90\end{array}$ & $\begin{array}{l}1,161.20 \\
1,281.20 \\
1,317.60 \\
1.319 .00 \\
1.342 .10\end{array}$ & $\begin{array}{l}08-11-92 \\
04-29-91 \\
05-02-91 \\
12-10-91 \\
01-20-92\end{array}$ & $367 C T T R$ \\
\hline & & & $\begin{array}{l}36.30 \\
37.40 \\
39.65 \\
39.10 \\
40.80\end{array}$ & $\begin{array}{l}1,343.70 \\
1,342.60 \\
1,340.35 \\
1,340.90 \\
1,339.20\end{array}$ & $\begin{array}{l}02-27-92 \\
03-17-92 \\
04-14-92 \\
05-14-92 \\
07-23-92\end{array}$ & \\
\hline \multirow[t]{2}{*}{39} & J IMMY BISHOP & $1,25 b$ & $\begin{array}{r}69.50 \\
97.00 \\
124.00 \\
83.85 \\
81.35\end{array}$ & $\begin{array}{l}1,310.50 \\
1,158.00 \\
1,131.00 \\
1,171.15 \\
1,173.65\end{array}$ & $\begin{array}{l}08-11-92 \\
05-07-91 \\
08-21-91 \\
11-19-91 \\
01-20-92\end{array}$ & $367 \mathrm{CTTR}$ \\
\hline & & & $\begin{array}{l}85.85 \\
86.25 \\
87.15 \\
86.65 \\
87.10\end{array}$ & $\begin{array}{l}1.169 .15 \\
1: 168.75 \\
1: 167.85 \\
1: 168.35 \\
1: 167.90\end{array}$ & $\begin{array}{l}02-27-92 \\
03-17-92 \\
04-14-92 \\
05-14-92 \\
07-23-92\end{array}$ & \\
\hline \multirow[t]{2}{*}{41} & JIMMY UAVIS & 1,240 & $\begin{array}{l}84.55 \\
64.50 \\
66.40 \\
79.80 \\
73.90\end{array}$ & $\begin{array}{l}1,170.45 \\
1.175 .50 \\
1.173 .60 \\
1.160 .20 \\
1.166 .10\end{array}$ & $\begin{array}{l}08-11-92 \\
05-07-91 \\
08-21-91 \\
11-19-91 \\
01-20-92\end{array}$ & $367 \mathrm{CTTR}$ \\
\hline & & & $\begin{array}{l}71.60 \\
74.40 \\
72.00 \\
71.50 \\
65.40\end{array}$ & $\begin{array}{l}1,168.40 \\
1,165.60 \\
1,168.00 \\
1,168.50 \\
1,174.60\end{array}$ & $\begin{array}{l}02-27-92 \\
03-17-92 \\
04-14-92 \\
05-14-92 \\
07-23-92\end{array}$ & \\
\hline \multirow[t]{2}{*}{42} & J IMMY DAVIS & 1.240 & $\begin{array}{l}66 \cdot 50 \\
63 \cdot 50 \\
65 \cdot 80 \\
72.90 \\
68.20\end{array}$ & $\begin{array}{l}1.173 .50 \\
1: 176.50 \\
1.174 .20 \\
1.167 .10 \\
1.171 .80\end{array}$ & $\begin{array}{l}08-11-92 \\
05-07-91 \\
08-21-91 \\
11-19-91 \\
01-20-92\end{array}$ & $367 \mathrm{CTTR}$ \\
\hline & & & $\begin{array}{l}66.10 \\
69.05 \\
68.00 \\
68.20 \\
66.30\end{array}$ & $\begin{array}{l}1.173 .90 \\
1,170.95 \\
1: 172.00 \\
1,171.80 \\
1,173.70\end{array}$ & $\begin{array}{l}02-27-92 \\
03-17-92 \\
04-14-92 \\
05-14-92 \\
07-23-92\end{array}$ & \\
\hline $\begin{array}{l}41 \\
49 \\
50 \\
51\end{array}$ & $\begin{array}{l}\text { HEN RATZLOFF } \\
\text { LARKY HARP } \\
\text { HARULU LOGAN } \\
\text { BEN RATZLOFF }\end{array}$ & $\begin{array}{l}1.290 \\
1,360 \\
1.240 \\
1,120\end{array}$ & $\begin{array}{r}68.10 \\
13.00 \\
3.00 \\
91.60 \\
8.30\end{array}$ & $\begin{array}{l}1.171 .90 \\
1.277 .00 \\
1,357.00 \\
1.148 .40 \\
1.111 .70\end{array}$ & $\begin{array}{l}08-11-92 \\
05-07-91 \\
05-09-91 \\
05-09-91 \\
05-09-91\end{array}$ & $\begin{array}{c}330 \text { BOON } \\
331 \text { BSVL } \\
364 \text { EVRN } \\
--\end{array}$ \\
\hline \multirow[t]{3}{*}{$\begin{array}{l}52 \\
56 \\
57 \\
58\end{array}$} & $\begin{array}{l}\text { BEN RATZLOFF } \\
\text { R.J HOLLAND } \\
\text { BUSTER POWELL } \\
\text { LONIIE USERV }\end{array}$ & $\begin{array}{l}1,110 \\
1,480 \\
1,420 \\
1,790\end{array}$ & $\begin{array}{r}40.80 \\
134.05 \\
12.30 \\
34.90 \\
37.55\end{array}$ & $\begin{array}{l}1.129 .10 \\
1.345 .95 \\
1.407 .70 \\
1.755 .10 \\
1.752 .45\end{array}$ & $\begin{array}{l}05-09-91 \\
05-15-91 \\
05-22-91 \\
05-28-91 \\
08-23-91\end{array}$ & $\begin{array}{l}367 C T T R \\
367 C T T R\end{array}$ \\
\hline & & & $\begin{array}{l}37.36 \\
36.20 \\
36.00 \\
35.90 \\
36.80\end{array}$ & $\begin{array}{l}1.752 .64 \\
1,753.80 \\
1.754 .00 \\
1.754 .10 \\
1.753 .20\end{array}$ & $\begin{array}{l}09-25-91 \\
12-10-91 \\
01-26-92 \\
02-27-92 \\
03-26-92\end{array}$ & \\
\hline & BOBEY ANDKEWS & 1,180 & $\begin{array}{l}36.60 \\
36.25 \\
37.00 \\
36.70 \\
11.40\end{array}$ & $\begin{array}{l}1.753 .40 \\
1.753 .75 \\
1.753 .00 \\
1.753 .30 \\
1.168 .60\end{array}$ & $\begin{array}{l}04-14-92 \\
05-14-92 \\
07-16-92 \\
08-11-92 \\
05-28-91\end{array}$ & $330800 \mathrm{~N}$ \\
\hline 59 & & & $\begin{array}{r}16.32 \\
8.30 \\
13.06 \\
13.05 \\
15.25\end{array}$ & $\begin{array}{l}1.163 .68 \\
1.171 .70 \\
1.166 .94 \\
1.166 .95 \\
1.164 .75\end{array}$ & $\begin{array}{l}08-23-91 \\
12-10-91 \\
01-21-92 \\
02-27-92 \\
04-14-92\end{array}$ & \\
\hline
\end{tabular}




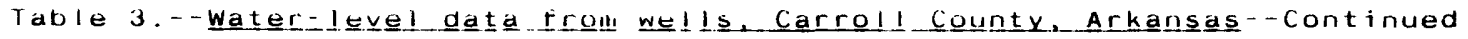

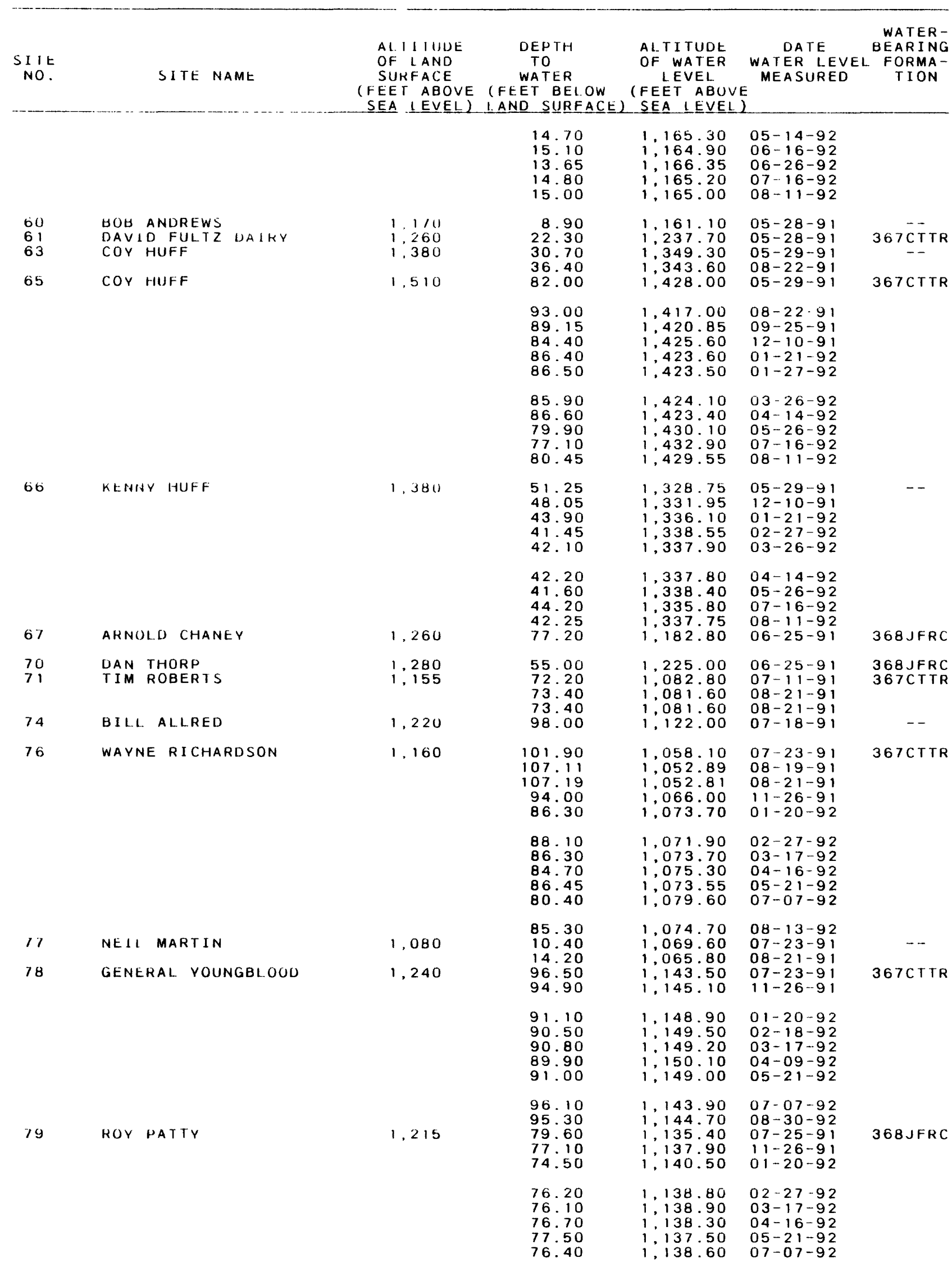




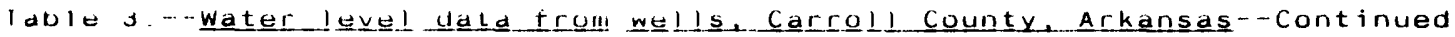

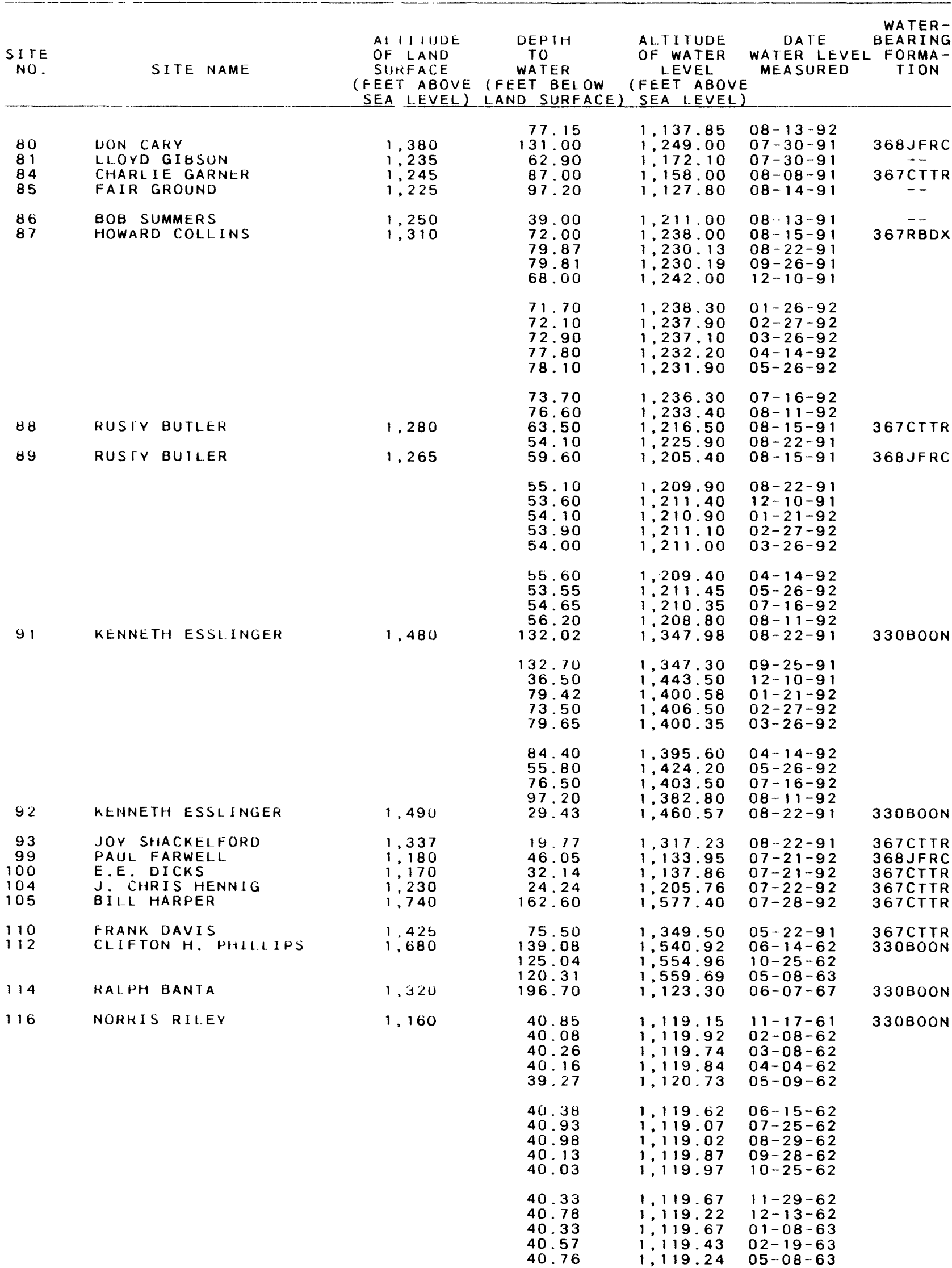


Table 3. Water level uata from wel ls. Corrol l Countye Arkansas--Continued

\begin{tabular}{|c|c|c|c|c|c|c|c|}
\hline $\begin{array}{c}S I I E \\
N \cap .\end{array}$ & SI & it NAME & $\begin{array}{l}\text { AL I I IUIDE } \\
\text { OF LAND } \\
\text { SUIRFACE } \\
\text { (FEET ABOVE } \\
\text { SEA IEVEL) }\end{array}$ & $\begin{array}{c}\text { DEPrH } \\
\text { TO } \\
\text { WATER } \\
(\text { FET BEI OW } \\
\text { LAND SU UREACEL) }\end{array}$ & $\begin{array}{l}\text { AL I I TUDE } \\
\text { OF WATER } \\
\text { I.EVEL } \\
\text { (FEET ABOVE } \\
\text { SEEA IEVEL) }\end{array}$ & $\begin{array}{l}\text { DATE } \\
\text { WATER I EVEL } \\
\text { MEASURED } \\
\end{array}$ & $\begin{array}{l}\text { WATER- } \\
\text { BEARING } \\
\text { FORMA- } \\
\text { TION }\end{array}$ \\
\hline $\begin{array}{lll}1 & 1 & 7 \\
1 & 1 & 8 \\
1 & 1 & 9 \\
1 & 2 & 1 \\
1 & 2 & 2\end{array}$ & \multicolumn{2}{|c|}{$\begin{array}{ll}\text { RICIIARD } & \text { HATILNHIAUR } \\
\text { GILBERT } & \text { RIVERA } \\
\text { US-CE } & \\
\text { MURIEL SCHMIDI } \\
\text { EUREKA SPRINGS CITY }\end{array}$} & $\begin{array}{l}1,662 \\
1: 400 \\
1: 172 \\
1: 525 \\
1: 400\end{array}$ & $\begin{array}{r}553.00 \\
208 \cdot 58 \\
77.85 \\
391.56 \\
273.00\end{array}$ & $\begin{array}{l}1,109.00 \\
1,191.42 \\
1.094 .15 \\
1.133 .44 \\
1,127.00\end{array}$ & $\begin{array}{l}08-06-76 \\
04-10-75 \\
05-14-73 \\
01-29-75 \\
07-06-72\end{array}$ & $\begin{array}{l}367 C T T R \\
367 R B D X \\
367 R B D X \\
367 R B D X \\
367 R B D X\end{array}$ \\
\hline \multirow[t]{3}{*}{123} & \multirow{3}{*}{\multicolumn{2}{|c|}{ EUREKA SPRINGS CITY }} & 1,290 & $\begin{array}{l}306.15 \\
342.75 \\
392.55 \\
429.63 \\
470.40\end{array}$ & $\begin{array}{l}989.85 \\
953 \cdot 25 \\
903.45 \\
866.37 \\
825.60\end{array}$ & $\begin{array}{l}08-26-76 \\
05-25-77 \\
05-03-78 \\
04-17-79 \\
03-26-80\end{array}$ & 371 POTS \\
\hline & & & & $\begin{array}{l}464.00 \\
432.35 \\
454.44 \\
287.43 \\
278.23\end{array}$ & $\begin{array}{r}832.00 \\
863.65 \\
841.56 \\
1.008 .57 \\
1.017 .77\end{array}$ & $\begin{array}{l}04-23-81 \\
05-11-82 \\
04-25-83 \\
04-04-84 \\
04-08-85\end{array}$ & \\
\hline & & & & $\begin{array}{l}274.31 \\
274.68 \\
272.10 \\
274.88 \\
276.20\end{array}$ & $\begin{array}{r}1,0221.69 \\
1,0221.32 \\
1.023 .90 \\
1,021.12 \\
1.019 .80\end{array}$ & $\begin{array}{l}04-14-86 \\
04-08-87 \\
05-06-88 \\
05-11-89 \\
03-28-90\end{array}$ & \\
\hline \multirow[t]{5}{*}{124} & \multirow{4}{*}{\multicolumn{2}{|c|}{ EUREKKA SPRINGS CITY }} & \multirow[t]{4}{*}{1.199 .60} & $\begin{array}{l}177.65 \\
197.80 \\
200.77 \\
201.80 \\
473.00\end{array}$ & $\begin{array}{r}1,021.95 \\
1,001.80 \\
998.83 \\
997.80 \\
726.60\end{array}$ & $\begin{array}{l}08-25-66 \\
11-02-66 \\
02-15-67 \\
06-07-67 \\
11-10-71\end{array}$ & \multirow[t]{5}{*}{367 GNTR } \\
\hline & & & & $\begin{array}{l}418.00 \\
392.00 \\
445.00 \\
417.82 \\
404.00\end{array}$ & $\begin{array}{l}781.60 \\
807.60 \\
754.60 \\
781.78 \\
795.60\end{array}$ & $\begin{array}{l}10-14-72 \\
11-29-73 \\
11-26-74 \\
05-25-75 \\
12-02-75\end{array}$ & \\
\hline & & & & $\begin{array}{l}424.44 \\
408.58 \\
438.30 \\
440.00 \\
208.75\end{array}$ & $\begin{array}{l}775.16 \\
791.02 \\
761.30 \\
759.60 \\
990.85\end{array}$ & $\begin{array}{l}05-03-78 \\
04-17-79 \\
03-26-80 \\
04-23-81 \\
05-20-81\end{array}$ & \\
\hline & & & & $\begin{array}{l}207.91 \\
208.58 \\
139.68 \\
124.58 \\
188.19\end{array}$ & $\begin{array}{r}991.69 \\
991.02 \\
1,059.92 \\
1,075.02 \\
1.011 .41\end{array}$ & $\begin{array}{l}06-10-82 \\
04-25-83 \\
04-04-84 \\
04-08-85 \\
04-15-86\end{array}$ & \\
\hline & HAU GR & IESENAUER & 1.520 & $\begin{array}{l}124.51 \\
185.62 \\
124.25 \\
125.82 \\
452.10\end{array}$ & $\begin{array}{r}1,075.09 \\
1,013.98 \\
1.075 .35 \\
1,073.78 \\
1,067.90\end{array}$ & $\begin{array}{l}04-08-87 \\
05-04-88 \\
05-11-89 \\
03-28-90 \\
03-31-77\end{array}$ & \\
\hline \multirow[t]{4}{*}{$\begin{array}{l}127 \\
128 \\
129 \\
130\end{array}$} & \multirow{4}{*}{\multicolumn{2}{|c|}{$\begin{array}{l}V . R \text { SOLBERY } \\
\text { HÜI. IUAY ISLAND } \\
\text { HOL I DAY ISLAND } \\
\text { HOL IDAY ISLAND }\end{array}$}} & \multirow[t]{4}{*}{$\begin{array}{l}1,550 \\
1,520 \\
1,500 \\
1,010\end{array}$} & $\begin{array}{r}133.60 \\
520.00 \\
146.00 \\
86.40 \\
110.77\end{array}$ & $\begin{array}{r}1,416.40 \\
1,000.00 \\
1,354.00 \\
923.60 \\
899.23\end{array}$ & $\begin{array}{l}06-20-68 \\
07-07-72 \\
06-30-77 \\
11-19-70 \\
11-10-71\end{array}$ & \multirow[t]{4}{*}{$\begin{array}{l}\text { 368JFRC } \\
\text { 367GNTR } \\
\text { 367RBDX } \\
\text { 367RBDX }\end{array}$} \\
\hline & & & & $\begin{array}{lll}1 & 1 & 7.19 \\
1 & 21.19 \\
1 & 21.19 \\
1 & 28.00 \\
1 & 06.50\end{array}$ & $\begin{array}{l}892.81 \\
888.81 \\
888.81 \\
882.00 \\
903.50\end{array}$ & $\begin{array}{l}10-19-72 \\
11-29-73 \\
11-26-74 \\
12-03-75 \\
05-23-77\end{array}$ & \\
\hline & & & & $\begin{array}{r}92.13 \\
96.87 \\
96.75 \\
105.00 \\
89.13\end{array}$ & $\begin{array}{l}917.87 \\
913.13 \\
913.25 \\
905.00 \\
920.87\end{array}$ & $\begin{array}{l}07-03-78 \\
04-17-79 \\
03-26-80 \\
04-23-81 \\
05-11-82\end{array}$ & \\
\hline & & & & $\begin{array}{r}136.14 \\
143.07 \\
134.10 \\
89.28 \\
90.87\end{array}$ & $\begin{array}{l}873.86 \\
866.93 \\
875.90 \\
920.72 \\
919.13\end{array}$ & $\begin{array}{l}04-25-83 \\
04-04-84 \\
04-08-85 \\
04-15-86 \\
04-08-87\end{array}$ & \\
\hline
\end{tabular}


Table 3. - water-level data from wel ls carroll County. Arkansas--Continued

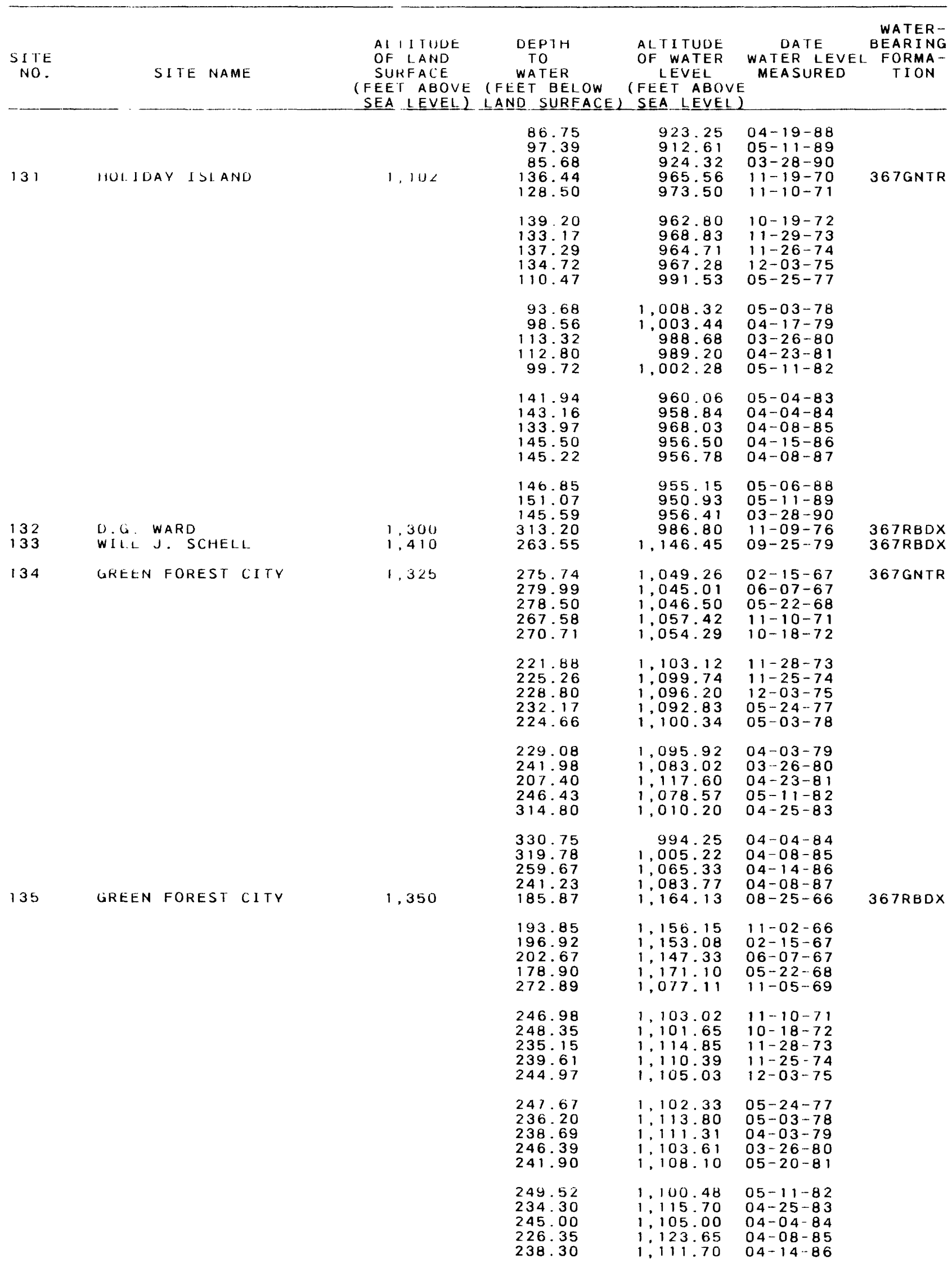


Table 3.--water level data from wells. Carroll County. Arkansas--Continued

\begin{tabular}{|c|c|c|c|c|c|c|}
\hline $\begin{array}{l}\text { SITE } \\
\text { NO. }\end{array}$ & SITE NAME & $\begin{array}{l}\text { AI I I IUUE } \\
\text { OF LAND } \\
\text { SUKFACE } \\
\text { (FEE ABOVE } \\
\text { SEA LEVEL) }\end{array}$ & $\begin{array}{c}\text { DEPTH } \\
\text { TO } \\
\text { WATER } \\
\text { (FEET BEL OW } \\
\text { LAND SUREACE) }\end{array}$ & $\begin{array}{c}\text { ALTITUDE } \\
\text { OF WATER } \\
\text { LEVEL } \\
\text { (FEET ABOVE } \\
\text { (FEA LEVEL) }\end{array}$ & $\begin{array}{l}\text { DATE } \\
\text { WATER LEVEL } \\
\text { MEASURED }\end{array}$ & $\begin{array}{l}\text { WATER- } \\
\text { BEARING } \\
\text { FORMA- } \\
\text { TION }\end{array}$ \\
\hline $\begin{array}{l}136 \\
137\end{array}$ & $\begin{array}{l}\text { LRR. K.A. ETHERINGIUN } \\
\text { REV. JOHN WYATT }\end{array}$ & $\begin{array}{l}1.525 \\
1.480\end{array}$ & $\begin{array}{l}244.50 \\
257.79 \\
250.17 \\
381.50 \\
462.00\end{array}$ & $\begin{array}{l}1,105.50 \\
1,092.21 \\
1,099.83 \\
1,143.50 \\
1,018.00\end{array}$ & $\begin{array}{l}05-05-88 \\
05-11-89 \\
03-28-90 \\
07-28-75 \\
03-09-77\end{array}$ & $\begin{array}{l}367 R B D X \\
368 J F R C\end{array}$ \\
\hline $\begin{array}{l}138 \\
146\end{array}$ & $\begin{array}{l}\text { ROGER A ANOERSON } \\
\text { MURL MALUNE }\end{array}$ & $\begin{array}{l}1.4011 \\
1.360\end{array}$ & $\begin{array}{r}451.00 \\
15.42 \\
15.29 \\
15.43 \\
15.47\end{array}$ & $\begin{array}{l}1,009.00 \\
1,345.00 \\
1,345.00 \\
1,345.00 \\
1,345.00\end{array}$ & $\begin{array}{l}03-08-77 \\
05-27-92 \\
06-03-92 \\
07-13-92 \\
08-25-92\end{array}$ & $\begin{array}{l}367 R B D X \\
330 B O O N\end{array}$ \\
\hline 141 & MUEL. MALUNE & $1=\frac{300}{0}$ & 26.98 & 1.333 .00 & $07-13-92$ & $330 B 0 O N$ \\
\hline
\end{tabular}




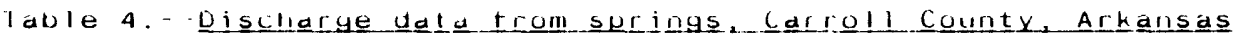

IC, Wl ent meter; E, estimated; V, volumetric; $331 B S V t$, Batesville Sandstone; 330BUON, Boone Formation; 367CTTR, Cotter Dolomite; <, less than]

\begin{tabular}{|c|c|c|c|c|c|c|}
\hline $\begin{array}{l}\text { SIIE } \\
\text { NO. }\end{array}$ & NAME OF SPRING & $\begin{array}{l}\text { AL I I TUDE } \\
\text { OF LAND } \\
\text { SURFACE } \\
\text { (FEET ABOVE } \\
\text { SEA LEVEL ) }\end{array}$ & $\begin{array}{l}\text { DISCHAKLIE } \\
\text { (GALLONS } \\
\text { PER MINUIEL }\end{array}$ & $\begin{array}{l}\text { DATE } \\
\text { DISCHARGE } \\
\text { MEASURED }\end{array}$ & $\begin{array}{l}\text { METHOD OF } \\
\text { DISCHARGE } \\
\text { MEASUREMENT }\end{array}$ & $\begin{array}{l}\text { STRATI- } \\
\text { GRAPHIC } \\
\text { HORIZON } \\
\text { OF SPRINC } \\
\text { ORIFICE }\end{array}$ \\
\hline 1 & BUNCH SPRING & 1.065 & $\begin{array}{l}180 \\
100\end{array}$ & $\begin{array}{l}10-29-90 \\
08-23-91\end{array}$ & $\begin{array}{l}C \\
E\end{array}$ & $\begin{array}{l}367 C T T R \\
367 C T T R\end{array}$ \\
\hline 2 & K. HAMM SPRING & 1.145 & & & $\begin{array}{l}E \\
E\end{array}$ & $\begin{array}{l}367 C T T R \\
367 C T T R\end{array}$ \\
\hline $\begin{array}{l}3 \\
6 \\
9\end{array}$ & $\begin{array}{l}\text { R.C. CHANEY SPRING } \\
\text { VERDA DOTSON SPRING } \\
\text { WINONA SPRING }\end{array}$ & $\begin{array}{l}1.230 \\
1.400 \\
1.320\end{array}$ & $\begin{array}{r}50 \\
1,200 \\
0.9\end{array}$ & $\begin{array}{l}10-29-90 \\
11-06-90 \\
09-26-91\end{array}$ & $\begin{array}{l}\text { E } \\
\mathrm{C}\end{array}$ & $\begin{array}{l}367 C T T R \\
330 B O O N \\
330 B O O N\end{array}$ \\
\hline \multirow[t]{5}{*}{10} & BI UE SPRING & 920 & $\begin{array}{l}4,600 \\
3,000 \\
4100 \\
2,900 \\
4,900\end{array}$ & $\begin{array}{l}06-29-51 \\
04-06-54 \\
09-01-54 \\
08-06-57 \\
06-12-58\end{array}$ & $\begin{array}{l}c \\
C \\
c \\
c \\
c\end{array}$ & $367 C T T R$ \\
\hline & & & $\begin{array}{l}1,800 \\
1,800 \\
1,100 \\
2,500 \\
1,600\end{array}$ & $\begin{array}{l}10-23-58 \\
08-11-59 \\
09-24-59 \\
08-24-60 \\
09-14-60\end{array}$ & $\begin{array}{l}\mathrm{C} \\
\mathrm{C} \\
\mathrm{C} \\
\mathrm{C} \\
\mathrm{C}\end{array}$ & \\
\hline & & & $\begin{array}{l}1,600 \\
3,500 \\
5,700 \\
2,600 \\
5,800\end{array}$ & $\begin{array}{l}10-13-60 \\
07-12-61 \\
09-13-61 \\
11-01-61 \\
05-22-62\end{array}$ & $\begin{array}{l}\mathrm{C} \\
\mathrm{C} \\
\mathrm{C} \\
\mathrm{C} \\
\mathrm{C}\end{array}$ & \\
\hline & & & $\begin{array}{l}3,400 \\
1,900 \\
1,300 \\
3,700 \\
1,300\end{array}$ & $\begin{array}{l}06-20-62 \\
07-19-62 \\
08-14-62 \\
04-18-63 \\
07-30-63\end{array}$ & $\begin{array}{l}\mathrm{C} \\
\mathrm{C} \\
\mathrm{C} \\
\mathrm{C} \\
\mathrm{C}\end{array}$ & \\
\hline & & & $\begin{array}{l}1,000 \\
1,900 \\
1,200 \\
1,100 \\
930\end{array}$ & $\begin{array}{l}12-09-65 \\
08-01-68 \\
09-03-87 \\
08-25-88 \\
10-06-88\end{array}$ & $\begin{array}{l}\mathrm{C} \\
\mathrm{C} \\
\mathrm{C} \\
\mathrm{C} \\
\mathrm{C}\end{array}$ & \\
\hline $\begin{array}{ll}1 & 7 \\
18 & 8 \\
21 & 1 \\
22 \\
23\end{array}$ & $\begin{array}{l}\text { WOHNSON SPKIHG } \\
\text { HALE SPRING } \\
\text { MCWILLIAMS SPRING } \\
\text { FLOYD SPRING } \\
\text { BERRYVILIE SPRING }\end{array}$ & $\begin{array}{r}1.490 \\
1.180 \\
1.370 \\
1.370 \\
1.178\end{array}$ & $\begin{array}{l}3.1 \\
480^{3} \\
10 \\
28\end{array}$ & 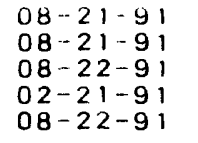 & $\begin{array}{l}E \\
C \\
E \\
E \\
E\end{array}$ & $\begin{array}{l}367 C T T R \\
330 B O O N \\
330 B O O N \\
330 B O O N \\
367 C T T R\end{array}$ \\
\hline $\begin{array}{l}26 \\
29 \\
43 \\
48 \\
64\end{array}$ & $\begin{array}{l}\text { BRASWELI SPRING } \\
\text { J.P RILEY SPRING } \\
\text { BASIN SPRING } \\
\text { HARP SPRING } \\
\text { COY HUFF SPRING }\end{array}$ & $\begin{array}{l}1,260 \\
1,200 \\
1,300 \\
1,310 \\
1,360\end{array}$ & $\begin{array}{l}50 \\
2.6 \\
13 \\
<.1 \\
280\end{array}$ & $\begin{array}{l}03-12-91 \\
08-21-91 \\
05-07-91 \\
07-09-92 \\
08-22-91\end{array}$ & $\begin{array}{l}E \\
E \\
E \\
E \\
C\end{array}$ & $\begin{array}{l}367 C T T R \\
367 C T T R \\
331 B S V L \\
330 B O O N \\
330 B O O N\end{array}$ \\
\hline $\begin{array}{l}72 \\
73 \\
82 \\
90 \\
94\end{array}$ & $\begin{array}{l}\text { WILSON SHRING } \\
\text { HALFIELD SPRING } \\
\text { ROBERTS SPRING } \\
\text { BUTLER SPRING } \\
\text { REEVES SPRING }\end{array}$ & $\begin{array}{l}1,390 \\
1,380 \\
1,090 \\
1,200 \\
1,130\end{array}$ & $\begin{aligned} 0^{.2} \\
150^{2} \\
3,000\end{aligned}$ & $\begin{array}{l}08-22-91 \\
07-15-91 \\
08-22-91 \\
08-22-91 \\
08-23-91\end{array}$ & $\begin{array}{l}E \\
E \\
E \\
E \\
C\end{array}$ & $\begin{array}{l}\text { 33OBOON } \\
330 B O O N \\
367 C T T R \\
367 C T T R \\
330 B O O N\end{array}$ \\
\hline $\begin{array}{l}109 \\
14 \underline{5} .\end{array}$ & $\begin{array}{l}\text { JUHINSON SPRING } \\
\text { MALONE SPRING }\end{array}$ & $\begin{array}{l}1,070 \\
1.340\end{array}$ & $\begin{array}{r}3.1 \\
350^{-1}\end{array}$ & $\begin{array}{l}O B-21-91 \\
05-12-92\end{array}$ & $\begin{array}{l}E \\
C\end{array}$ & $\begin{array}{l}367 C T T R \\
330 \text { OOON }\end{array}$ \\
\hline
\end{tabular}


Table 5. - Luricentratiuns of majur constituents and properties

IMS/cm, micrusiemens per centimeter at 25 degrees Celsius; ${ }^{\circ} \mathrm{C}$, degiees Celsius; mg/L, milligrams per

\begin{tabular}{|c|c|c|c|c|c|c|c|c|}
\hline $\begin{array}{l}\text { SIIE } \\
\text { NO. }\end{array}$ & - SIIt NAME & $\begin{array}{l}\text { LOCAL } \\
\text { WELL } \\
\text { NO. }\end{array}$ & DATE & $\begin{array}{l}\text { SPE } \\
\text { CIFIC } \\
\text { CON- } \\
\text { DUCT- } \\
\text { ANCE } \\
(\mu S / C I I)\end{array}$ & $\begin{array}{l}\text { PH } \\
\text { (STAND - } \\
\text { ARD } \\
\text { UNIIS) }\end{array}$ & $\begin{array}{l}\text { TEMPER- } \\
\text { ATURE } \\
\text { WATER } \\
\left.\text { ( }^{\circ} \mathrm{C}\right)\end{array}$ & $\begin{array}{l}\text { COLOR } \\
\text { (PLAT- } \\
\text { INUM- } \\
\text { COBALT } \\
\text { UNITS) }\end{array}$ & $\begin{array}{l}\text { HARD- } \\
\text { NESS } \\
\text { TOTAL } \\
\text { (mg/L } \\
\text { as } \\
\text { CaCO3) }\end{array}$ \\
\hline $\begin{array}{r}1 \\
8 \\
9 \\
18 \\
31\end{array}$ & $\begin{array}{l}\text { BUNCH SFRING } \\
\text { KENNEIH CLARK } \\
\text { WINONA SPRING } \\
\text { HALE SHRING } \\
\text { WAYNE HARNESS }\end{array}$ & $\begin{array}{l}\text { 20N25W26DBD1SP } \\
\text { 19N24W05ADD1 } \\
\text { 19N25WO6CDD1SP } \\
21 N 23 W 17 D D A 1 S P \\
21 N 23 W 17 A A B 1\end{array}$ & $\begin{array}{l}09-26-91 \\
09-26-91 \\
09-26-91 \\
09-24-91 \\
09-24-91\end{array}$ & $\begin{array}{l}478 \\
402 \\
163 \\
369 \\
428\end{array}$ & $\begin{array}{l}7.2 \\
7.6 \\
7.8 \\
7.2 \\
7.4\end{array}$ & $\begin{array}{l}15.0 \\
15.5 \\
14.0 \\
14.0 \\
15.5\end{array}$ & $\begin{array}{l}2 \\
1 \\
2 \\
2 \\
5\end{array}$ & $\begin{array}{r}270 \\
220 \\
89 \\
170 \\
230\end{array}$ \\
\hline $\begin{array}{l}34 \\
39 \\
58 \\
64 \\
65\end{array}$ & $\begin{array}{l}\text { BUDDY UAVIDSON } \\
\text { JIMMY BISHOP } \\
\text { LONNIE USERY } \\
\text { COY HUFF SPRING } \\
\text { COY HUFF }\end{array}$ & $\begin{array}{l}21 N 25 W 25 A C C 1 \\
20 N 23 W 12 C C B 1 \\
19 N 22 W 34 B C B 1 \\
18 N 23 W 25 C D A 1 \triangle P \\
18 N 23 W 26 D A A 1\end{array}$ & $\begin{array}{l}09-24-91 \\
09-24-91 \\
09-25-91 \\
09-25-91 \\
09-25-91\end{array}$ & $\begin{array}{l}498 \\
435 \\
521 \\
358 \\
677\end{array}$ & $\begin{array}{l}7.9 \\
7.5 \\
7.2 \\
7.2 \\
7.0\end{array}$ & $\begin{array}{l}16.5 \\
16.5 \\
15.5 \\
16.0 \\
16.0\end{array}$ & $\begin{array}{r}1 \\
- \\
2 \\
5 \\
2\end{array}$ & $\begin{array}{r}260 \\
-- \\
260 \\
180 \\
370\end{array}$ \\
\hline $\begin{array}{l}87 \\
90 \\
91 \\
94 \\
95\end{array}$ & $\begin{array}{l}\text { HOWAKD COLLINS } \\
\text { BUTIER SPRING } \\
\text { KENNETII ESSLINGER } \\
\text { REEVES SPRING } \\
\text { CLIFFOKD BOETTNER }\end{array}$ & 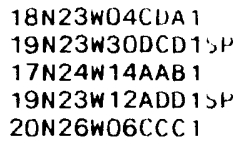 & $\begin{array}{l}09-26-91 \\
09-26-91 \\
09-25-91 \\
09-23-91 \\
09-27-91\end{array}$ & $\begin{array}{l}407 \\
405 \\
406 \\
568 \\
718\end{array}$ & $\begin{array}{l}7.5 \\
7.3 \\
7.4 \\
6.9 \\
7.2\end{array}$ & $\begin{array}{l}16.0 \\
16.5 \\
15.5 \\
17.0 \\
16.0\end{array}$ & $\begin{array}{r}2 \\
-- \\
2 \\
25 \\
--\end{array}$ & $\begin{array}{r}220 \\
-- \\
220 \\
180 \\
--\end{array}$ \\
\hline $\begin{array}{l}123 \\
124\end{array}$ & $\begin{array}{ll}\text { EUREKA SPRINGS CITY } \\
\text { EUREKA SPRINGS CIIY }\end{array}$ & $\begin{array}{l}\text { 2ON26W23ACA } 1 \\
20 N 26 W 16 D C A 1\end{array}$ & $\begin{array}{l}09-01-76 \\
07-28-54 \\
09-01-75\end{array}$ & $\begin{array}{l}- \\
-- \\
--\end{array}$ & $\begin{array}{l}8.4 \\
-8.2\end{array}$ & $\begin{array}{l}-- \\
-- \\
--\end{array}$ & $\begin{array}{l}\cdots \\
-- \\
--\end{array}$ & $\begin{array}{l}140 \\
170 \\
150\end{array}$ \\
\hline 126 & DEWAYNE TIPTON & 20N22WO3DDA 1 & $\begin{array}{l}06-20-87 \\
09-17-87\end{array}$ & $\begin{array}{l}418 \\
480\end{array}$ & $\begin{array}{l}7.5 \\
7.0\end{array}$ & $\begin{array}{l}22.0 \\
17.0\end{array}$ & -- & $\begin{array}{l}220 \\
220\end{array}$ \\
\hline $\begin{array}{r}130 \\
131 \\
135 \\
\end{array}$ & $\begin{array}{l}\text { HOL I DAY I SLAND } \\
\text { HOL IDAY ISLAND } \\
\text { GREEN FOREST CITY }\end{array}$ & $\begin{array}{l}21 N 26 W 17 B C C 1 \\
21 N 26 W 15 B A A 1 \\
19 N 23 W 04 B A C 1\end{array}$ & $\begin{array}{l}08 \cdot 01-76 \\
08-02-73 \\
08-01-76 \\
03-01-65 \\
\end{array}$ & $\begin{array}{l}-- \\
-- \\
-- \\
--\end{array}$ & $\begin{array}{r}8.3 \\
- \\
-8.4 \\
--\end{array}$ & $\begin{array}{ll}\cdots \\
\cdots \\
-- \\
--\end{array}$ & $\begin{array}{r}-- \\
5 \\
-- \\
1\end{array}$ & $\begin{array}{l}280 \\
230 \\
230 \\
250 \\
\end{array}$ \\
\hline
\end{tabular}


in water trom selected wells and surings ill Carroll County. Arkansas

liter; -.- not determined; <, signifies concentration is below detection limit of the value specified]

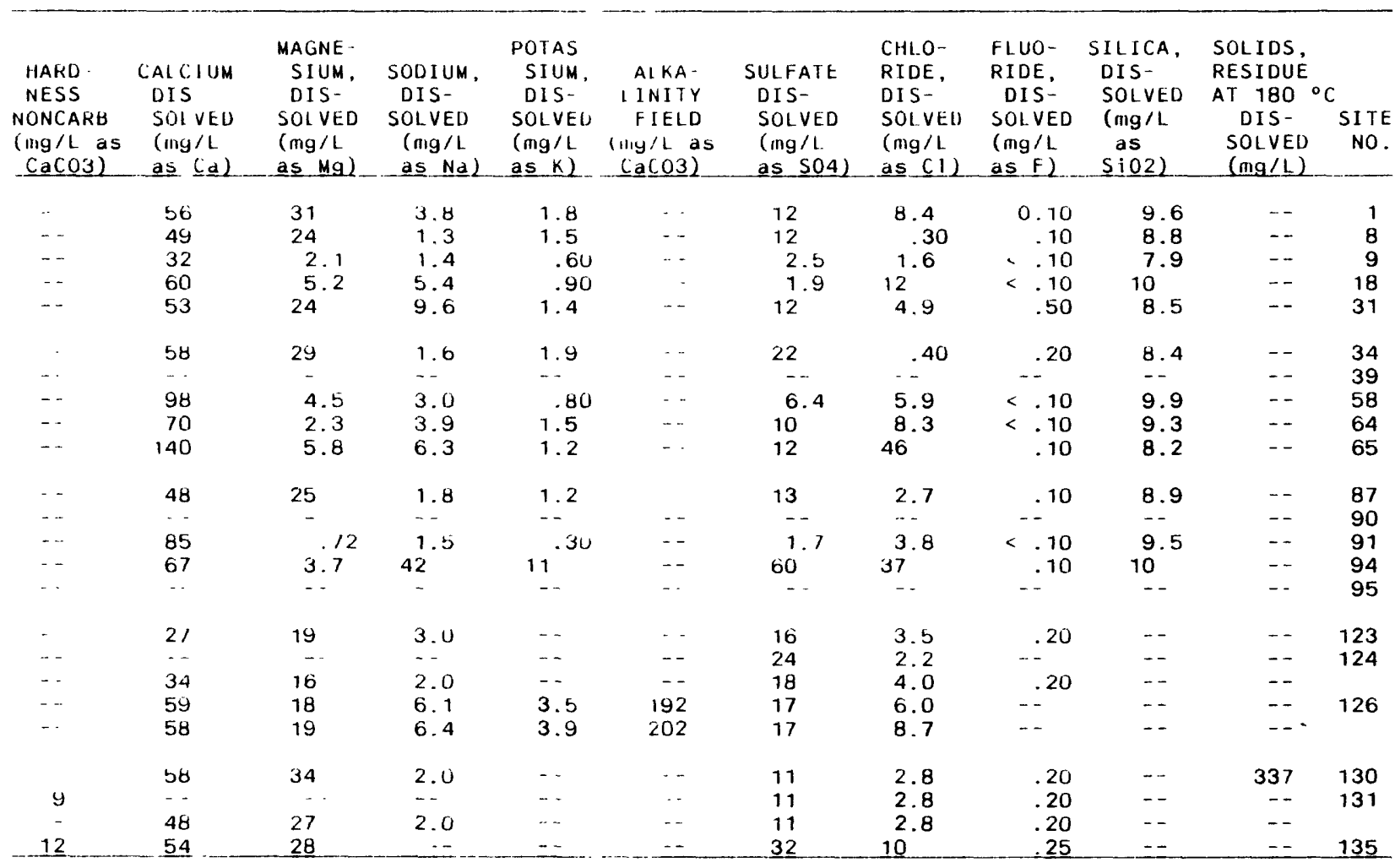


Table o. - Concentratiolls uf trace constituents in water from L $\mu \mathrm{g} / \mathrm{L}$, micrugrams per liter; <, signifies concentration is below

\begin{tabular}{|c|c|c|c|c|c|c|c|c|c|}
\hline $\begin{array}{l}\text { SITt } \\
\text { NO. }\end{array}$ & SIIE NAME & $\begin{array}{l}\text { LOCAL } \\
\text { WELL } \\
\text { NO. }\end{array}$ & DA IE & $\begin{array}{l}\text { ALUM- } \\
\text { INUM, } \\
\text { DIS- } \\
\text { SOLVED } \\
(\mu g / L \\
\text { aS AI) }\end{array}$ & $\begin{array}{c}\text { ARSENIC } \\
\text { DIS- } \\
\text { SOLVED } \\
(\mu g / L \\
\text { as As })\end{array}$ & $\begin{array}{l}\text { BARIUM, } \\
\text { DIS- } \\
\text { SOLVED } \\
(\mu g / L . \\
\text { as Ba) }\end{array}$ & $\begin{array}{l}\text { BERYL- } \\
\text { LIUM, } \\
\text { DIS - } \\
\text { SOLVED } \\
(\mu g / L \\
\text { as Be) }\end{array}$ & $\begin{array}{c}\text { CADMIUM } \\
\text { DIS- } \\
\text { SOLVED } \\
(\mu g / L \\
\text { as Cd) }\end{array}$ & $\begin{array}{l}\text { CHRO- } \\
\text { MIUM, } \\
\text { DIS- } \\
\text { SOLVED } \\
(\mu g / L \\
\text { as Cr) }\end{array}$ \\
\hline 1 & BUNCH SPRING & $20 N 25 W 260 B D 1 S P$ & $0 y-26 \cdot 91$ & $<10$ & $<1$ & 42 & -0.5 & $<1.0$ & $<1$ \\
\hline 8 & KENNETH CLARK & 19N24W05ADD1 & $09-2691$ & $<10$ & $<1$ & 7 & $<.5$ & $<1.0$ & $<1$ \\
\hline 9 & WINONA SPRING & $19 N 25 W 06 C D D 1 S P$ & $09-26-91$ & 10 & $<1$ & 16 & $<.5$ & $<1.0$ & $<1$ \\
\hline 18 & HALE SPRING & $21 N 23 W 17 D D A 1 S P$ & $09-24-91$ & 10 & $<1$ & 28 & $<.5$ & $<1.0$ & 1 \\
\hline 31 & WAYNE HARNESS & $21 N 23 W 17 A A B 1$ & $0 y-24-91$ & 20 & 2 & 17 & $<.5$ & $<1.0$ & $<1$ \\
\hline 34 & BUUDY UAVIDSON & $21 N 25 W 25 A C C 1$ & $0: 92491$ & 10 & $<1$ & 23 & -.5 & $<1.0$ & $<1$ \\
\hline 58 & LONNIE USERY & $19 N 22 W 34 B C B 1$ & $09-2591$ & 20 & $<1$ & 26 & $<.5$ & $<1.0$ & $<1$ \\
\hline 64 & COY HUFF SPRING & $18 N 23 W 25 C D A 1 S P$ & $09-25 \cdot 91$ & 20 & $<1$ & 43 & $<.5$ & $<1.0$ & $<1$ \\
\hline 65 & COY HUFF & 18 N23W26DAA 1 & $0 y-25-91$ & 20 & $<1$ & 51 & $<.5$ & $<1.0$ & $<1$ \\
\hline 87 & HOWARD COLLINS & $18 N 23 W 04 C D A 1$ & $09-2691$ & 10 & $<1$ & 10 & $<.5$ & $<1.0$ & $<1$ \\
\hline 91 & KENNEIH ESSLINGER & $17 N 24 W 14 A A B 1$ & (u) 2591 & 10 & $\cdot 1$ & 21 & $<.5$ & $<1.0$ & $<1$ \\
\hline 94 & REEVES SPRING & 19N23W12ADD1SH & $0 y-23-91$ & 20 & $<1$ & 46 & $<.5$ & $<1.0$ & $<1$ \\
\hline 126 & DEWAYNE TIPTON & $20 N 22$ WO3DDA 1 & $00-20-87$ & -- & -- & - & -- & $<.6$ & -- \\
\hline & & & $09-17-87$ & $\ldots$ & $\ldots$ & -- & -- & 1.0 & -- \\
\hline 131 & HOL IDAY ISLAND & $21 N 26 W 15 B A A 1$ & $08-02-73$ & 200 & $\ldots$ & $\ldots$ & -- & $\ldots$ & 10 \\
\hline
\end{tabular}


selected wells and springs in Carroll County. Arkansas

delection limit of the value specified; - , not determined

\begin{tabular}{|c|c|c|c|c|c|c|c|c|c|c|c|}
\hline $\begin{array}{l}\text { COPPER } \\
\text { DIS- } \\
\text { SOIVED } \\
(\mu g / L \\
\text { as Cu) }\end{array}$ & $\begin{array}{c}\text { IRON, } \\
\text { DIS } \\
\text { SOLVEO } \\
(\mu g / 1 \\
\text { as Fe) }\end{array}$ & $\begin{array}{c}\text { LEAD, } \\
\text { DIS- } \\
\text { SOLVED } \\
(\mu g / L . \\
\text { as PD) }\end{array}$ & $\begin{array}{l}\text { MERCURY } \\
\text { DIS- } \\
\text { SOLVED } \\
(\mu g / L \\
\text { as HgL }\end{array}$ & $\begin{array}{c}\text { MOLYB } \\
\text { DENUM, } \\
\text { DIS- } \\
\text { SOLVED } \\
(\mu g / L \\
\text { as MO) }\end{array}$ & $\begin{array}{l}\text { NICREL, } \\
\text { DIS- } \\
\text { SOI VED } \\
(\mu g / L \\
\text { aS Ni) }\end{array}$ & $\begin{array}{c}\text { SELE- } \\
\text { NIUM, } \\
\text { DIS- } \\
\text { SOLVED } \\
\text { ( } \mu g / L \\
\text { as Se) }\end{array}$ & $\begin{array}{c}\text { SILVER, } \\
\text { DIS- } \\
\text { SOLVED } \\
(\mu g / L \\
\text { aS } \mathrm{Ag}) .\end{array}$ & $\begin{array}{c}\text { STRON- } \\
\text { TIUM, } \\
\text { DIS- } \\
\text { SOLVED } \\
(\mu g / L \\
\text { as Sr) }\end{array}$ & $\begin{array}{l}\text { VANA- } \\
\text { DIUM, } \\
\text { DIS- } \\
\text { SOLVED } \\
(\mu g / L \\
\text { as V) }\end{array}$ & $\begin{array}{c}\text { ZINC, } \\
\text { DIS- } \\
\text { SOLVED } \\
(\mu g / L \\
\text { as } Z n)\end{array}$ & $\begin{array}{c}\text { SITE } \\
\text { NO. }\end{array}$ \\
\hline$<1$ & $<3$ & $<1$ & 0.1 & $<1$ & -1 & $<1$ & $<1.0$ & 46 & $<1$ & 10 & 1 \\
\hline$<1$ & 31 & $<1$ & 1 & 9 & $\therefore 1$ & $<1$ & $<1.0$ & 45 & $<1$ & 14 & 8 \\
\hline$<1$ & $<3$ & $<1$ & .1 & $<1$ & $<1$ & $<1$ & $<1.0$ & 24 & $<1$ & 3 & 9 \\
\hline$<1$ & 6 & $<1$ & .1 & $<1$ & $<1$ & $<1$ & $<1.0$ & 32 & $<1$ & 16 & 18 \\
\hline$<1$ & 97 & $<1$ & .1 & $<1$ & $<1$ & $<1$ & $<1.0$ & 260 & $<1$ & 510 & 31 \\
\hline$<1$ & 39 & $<1$ & .1 & 1 & $<1$ & $<1$ & $<1.0$ & 48 & $<1$ & 180 & 34 \\
\hline 2 & 10 & $<1$ & .1 & $<1$ & $<1$ & $<1$ & $<1.0$ & 74 & $<1$ & 9 & 58 \\
\hline$<1$ & 6 & $<1$ & .1 & $<1$ & $<1$ & $<1$ & $<1.0$ & 64 & $<1$ & 5 & 64 \\
\hline 1 & 9 & 1 & .1 & 1 & $<1$ & $<1$ & $<1.0$ & 170 & $<1$ & 59 & 65 \\
\hline 3 & 10 & $<1$ & .1 & 6 & $<1$ & $<1$ & $<1.0$ & 69 & $<1$ & 46 & 87 \\
\hline 2 & 6 & $<1$ & .1 & $<1$ & $<1$ & $<1$ & $<1.0$ & 38 & $<1$ & 210 & 91 \\
\hline 3 & 13 & $<1$ & .1 & $<1$ & 2 & $<1$ & $<1.0$ & 79 & 2 & 10 & 94 \\
\hline$<3$ & 4 & 10 & $\therefore$ & -- & -6 & $\cdots$ & $\therefore$ & - & $=$ & 630 & 126 \\
\hline$<2$ & 8 & $<2$ & -- & -- & $<5$ & -- & - & -- & -- & 290 & \\
\hline 100 & $\ldots$ & 10 & 1.0 & -- & $\cdots$ & $=-$ & 10 & -- & $=-$ & 100 & 131 \\
\hline
\end{tabular}


Tatle 7. - concentration of nutrients and microorganisms in water from! selected wel Is and springs in Carroll County. Arkansas

[mg/L, milligrams per I iter; cols., colonies; mL, milliliters; <, signifies concentration is below detection limit of the value specified; --, not determined; $k$, plate count outside ideal rangel

\begin{tabular}{|c|c|c|c|c|c|c|c|c|}
\hline $\begin{array}{l}\text { SIIE } \\
\text { NOE. }\end{array}$ & SIIE NAME & $\begin{array}{l}1 O C A L \\
\text { WELL } \\
\text { NO. }\end{array}$ & DAIE & $\begin{array}{l}\text { NITRO- } \\
\text { GEN, } \\
\text { AMMONIA } \\
\text { DIS- } \\
\text { SOIVED } \\
\text { (mg/L } \\
\text { dS N) }\end{array}$ & $\begin{array}{l}\text { NLIRO- } \\
\text { GEN, } \\
\text { NU2+NO3 } \\
\text { DIS- } \\
\text { SOLVEO } \\
(\mathrm{mg} / L \\
\text { aS NL) }\end{array}$ & $\begin{array}{l}\text { PHOS- } \\
\text { PHORUS } \\
\text { ORTHO, } \\
\text { DIS- } \\
\text { SOLVED } \\
\text { (mg/L } \\
\text { as P) }\end{array}$ & $\begin{array}{l}\text { COLI- } \\
\text { FORM, } \\
\text { FECAL, } \\
\text { MF AGAR } \\
\text { (cols. per } \\
100 \text { inL) }\end{array}$ & $\begin{array}{l}\text { STREP- } \\
\text { TOCOCCI } \\
\text { FECAL. } \\
\text { KF AGAR } \\
\text { (COls. } \\
\text { per } \\
100 \mathrm{~mL})\end{array}$ \\
\hline $\begin{array}{r}1 \\
8 \\
9 \\
18 \\
23\end{array}$ & $\begin{array}{l}\text { BUNCH SIPING } \\
\text { KENNEIH CLARK } \\
\text { WINONA SPRING } \\
\text { HALE SPRING } \\
\text { BERRYVILLE SPRING }\end{array}$ & $\begin{array}{l}20 N 25 W 26 D B D 1 S P \\
19 N 24 W 05 A D D 1 \\
19 N 25 W 06 C D D 1 S P \\
21 N 23 W 17 D D A 1 S P \\
20 N 24 W 30 D B A 1 S P\end{array}$ & $\begin{array}{l}09-26-91 \\
09-26-91 \\
09-26-91 \\
09-24-91 \\
09-27-91\end{array}$ & $\begin{array}{r}0.010 \\
.020 \\
<\quad .010 \\
<.010 \\
.-\end{array}$ & $\begin{aligned} & 1.20 \\
< & .050 \\
< & .050 \\
2.00 & --\end{aligned}$ & $\begin{array}{l}0.020 \\
<0.010 \\
<0.010 \\
0.020 \\
--\end{array}$ & $\begin{array}{r}63 \\
0 \\
0 \\
22 \\
110\end{array}$ & $\begin{array}{r}1,000 \\
0 \\
82 \\
190 \\
51\end{array}$ \\
\hline $\begin{array}{l}31 \\
34 \\
39 \\
58 \\
64\end{array}$ & $\begin{array}{l}\text { WAYNE HARNESS } \\
\text { BUDOY DAVIDSON } \\
\text { JIMMY BISHOP } \\
\text { LONNIE USERY } \\
\text { COY HUFF SPRING }\end{array}$ & $\begin{array}{l}21 N 23 W 17 A A B 1 \\
21 N 25 W 25 A C C 1 \\
20 N 23 W 12 C C B 1 \\
19 N 22 W 34 B C B 1 \\
18 N 23 W 25 C D A 15 P\end{array}$ & $\begin{array}{l}09-24-91 \\
09-24-91 \\
09-24-91 \\
09-25-91 \\
09-25-91\end{array}$ & $\begin{array}{l}.010 \\
.010 \\
- \\
.010 \\
-.010\end{array}$ & $\begin{array}{l}.290 \\
<.050 \\
-. \\
2.90 \\
1.40\end{array}$ & $\begin{array}{c}<0.010 \\
<0.010 \\
- \\
<0.010 \\
<0.010\end{array}$ & $\begin{array}{r}0 \\
0 \\
0 \\
0 \\
K 61\end{array}$ & $\begin{array}{r}0 \\
0 \\
0 \\
0 \\
240\end{array}$ \\
\hline $\begin{array}{l}65 \\
87 \\
90 \\
91 \\
94\end{array}$ & $\begin{array}{l}\text { COY HUFF } \\
\text { HOWARD COLLINS } \\
\text { BUTLER SPRING } \\
\text { KENNETH ESSLINGER } \\
\text { REEVES SPRING }\end{array}$ & $\begin{array}{l}\text { 18N23W26DAA } 1 \\
18 N 23 W 04 C D A 1 \\
19 N 23 W 30 D C D 1 S P \\
17 N 24 W 14 A A B 1 \\
19 N 23 W 12 A D D 1 S P\end{array}$ & $\begin{array}{l}09-25-91 \\
09-26-91 \\
09-26-91 \\
09-25-91 \\
09-23-91\end{array}$ & $\begin{array}{l}.010 \\
<.010 \\
. \\
<.010 \\
<.010\end{array}$ & $\begin{array}{l}.880 \\
-.050 \\
- \\
.820 \\
7.20\end{array}$ & $\begin{array}{c}<0.010 \\
<0.010 \\
-. \\
<0.010 \\
0.540\end{array}$ & $\begin{array}{r}0 \\
0 \\
130 \\
0 \\
K 1,000\end{array}$ & $\begin{array}{r}150 \\
0 \\
140 \\
0 \\
K 7,300\end{array}$ \\
\hline $\begin{array}{r}95 \\
126\end{array}$ & $\begin{array}{l}\text { CLIFFORU BOETINER } \\
\text { DEWAYNE IIPTON }\end{array}$ & $\begin{array}{l}20 N 26 \text { WO6CCC } 1 \\
20 N 22 \text { W03DDA } 1\end{array}$ & $\begin{array}{l}09-27-91 \\
06-20-87 \\
09-17-87\end{array}$ & .050 & $\begin{array}{l}-- \\
-- \\
--\end{array}$ & $\begin{array}{l}0.010 \\
0.010\end{array}$ & $\begin{array}{l}0 \\
0 \\
0\end{array}$ & $\begin{array}{l}7 \\
1 \\
2 \\
\end{array}$ \\
\hline
\end{tabular}


Iable 6. - Identification of gellera! aleds of sinkholes dnd internd I y drained. closed depressions identifiable on 7.5-minute topographic guadrangle maps in Carroll County, Arkansas

\begin{tabular}{|c|c|c|c|c|c|c|c|}
\hline $\begin{array}{l}\text { Site } \\
\text { llumber }\end{array}$ & Gene & eral a & areas of occurrence & $\begin{array}{l}71 / 2 \text { minute } \\
\text { topographic } \\
\text { quadrangle }\end{array}$ & $\begin{array}{l}\text { Geologic forma- } \\
\text { tion in which } \\
\text { depression occurs }\end{array}$ & $\begin{array}{l}\text { Altitude } \\
\text { of bottom of } \\
\text { depression } \\
\text { (feet above } \\
\text { sea level) }\end{array}$ & $\begin{array}{l}\text { Number } \\
\text { of } \\
\text { closed } \\
\text { depressioris }\end{array}$ \\
\hline 501 & T21N & R26W & section 14 & Eureka Springs & Cotter Dolomite & 1,180 & 1 \\
\hline 502 & T21N & R23W & sections $13,14,15,22,23$ & Blue Eye & Boone formation & $1,080-1,180$ & 4 \\
\hline 503 & T21N & R23W & section 30 & Blue Eye & Buone Formation & $1,320-1,340$ & 4 \\
\hline 504 & T21N & R22W & sections 34,27 & Denver & Boone Formation & $1,140 \cdot 1,260$ & 7 \\
\hline bU5 & $\mathrm{T} 21 \mathrm{~N}$ & $R 22 W$ & section 32 & Leriver & Boune Forllation & 1,160 & 2 \\
\hline 506 & $\begin{array}{l}\text { T } 21 \mathrm{~N} \\
\text { T } 20 \mathrm{~N}\end{array}$ & $\begin{array}{l}\text { R23W } \\
\text { R23W }\end{array}$ & $\begin{array}{l}\text { section } 33 \\
\text { sections } 4,5\end{array}$ & Hlue Eye & Boune for llation & $1,240-1,260$ & 3 \\
\hline 507 & $\begin{array}{l}\text { T } 20 N \\
\text { T } 20 N\end{array}$ & $\begin{array}{l}\text { R24W } \\
\text { R23W }\end{array}$ & $\begin{array}{l}\text { sections } 13,24 \\
\text { sections } 7,8,17,18,19\end{array}$ & Blue Eye & Buont formation & $1,280-1,440$ & 5 \\
\hline sut & $120 \mathrm{~N}$ & $k 22 w$ & sections 7,18 & Leriver & Buone formation & $1,220-1,240$ & 3 \\
\hline 509 & T $20 N$ & $k 22 W$ & sections 8,17 & Denver & Hoone Formation & $1,140-1,180$ & 2 \\
\hline 510 & $1 \angle O N$ & k23w & sections $14,15,21,22,23,28$ & $\begin{array}{l}\text { Blue Eye, } \\
\text { Green Forest }\end{array}$ & Batesville Sandstone & $1,260 \cdot 1,330$ & 11 \\
\hline b11 & $120 N$ & $\mathrm{~K} 22 \mathrm{~W}$ & section 20 & Denver & Boone Formation & $1,100-1,180$ & 3 \\
\hline 512 & $\mathrm{~T} 2 \mathrm{ON}$ & $\mathrm{k} 23 \mathrm{~W}$ & section 29 & Green Forest & Batesville Sandstone & $1,220-1,320$ & 2 \\
\hline 513 & T2ON & $R 23 W$ & sectiun 29 & Alpena & Boone formation & $1,200-1,280$ & 2 \\
\hline 514 & $\begin{array}{l}120 N \\
\text { T } 19 N\end{array}$ & $\begin{array}{l}\text { R2.3W } \\
\text { R23W }\end{array}$ & $\begin{array}{l}\text { sections } 30,31,32 \\
\text { section } 5\end{array}$ & Green forest & Batesville Sanustone & $1,280-1,360$ & 5 \\
\hline 515 & $\begin{array}{l}120 N \\
T 19 N\end{array}$ & $\begin{array}{l}R 22 W \\
R 22 W\end{array}$ & $\begin{array}{l}\text { sections } 27,28,29,33,34 \\
\text { sections } 10,3\end{array}$ & Alpena & Buone formation & $1,120-1,300$ & 11 \\
\hline 516 & T 19N & $R 24 W$ & sections $4,5,8,9$ & Berryville & Cutter Dolonite & $1,120-1,220$ & 5 \\
\hline 517 & T $19 N$ & $k 23 w$ & section 6 & Green forest & Boorie formation & 1,400 & 2 \\
\hline 518 & $119 \mathrm{~N}$ & K23w & sections $y, 1 u$ & Green Forest & Batesville Sandstone & $1,280-1,320$ & 3 \\
\hline 519 & $119 N$ & $\mathrm{R} 23 \mathrm{~W}$ & sections 2,3 & Green forest & Batesville Sandstone & $1,300-1,330$ & 2 \\
\hline 520 & $\begin{array}{l}\text { T } 19 N \\
\text { T } 19 N\end{array}$ & $\begin{array}{l}\text { R22W } \\
\text { R23W }\end{array}$ & $\begin{array}{l}\text { section } \\
\text { section } 1\end{array}$ & $\begin{array}{l}\text { lireen forest, } \\
\text { Alpena }\end{array}$ & Boone Formation & $1,240-1,260$ & 3 \\
\hline 521 & $\begin{array}{l}\text { T2UN } \\
\text { T19N }\end{array}$ & $\begin{array}{l}\text { R22W } \\
\text { R22W }\end{array}$ & $\begin{array}{l}\text { section } 33 \\
\text { sections } 4,5,8,10\end{array}$ & Alpena & Boone formation & $1,200-1,320$ & 12 \\
\hline 522 & $\mathrm{~T} 19 \mathrm{~N}$ & $\mathrm{R} 23 \mathrm{~W}$ & sections 24,25 & ureen forest & Batesville Sandstone & $1,320-1,340$ & 2 \\
\hline 523 & $\begin{array}{l}\text { T19N } \\
\text { T19N }\end{array}$ & $\begin{array}{l}\mathrm{R} 23 \mathrm{~W} \\
\mathrm{R} 22 \mathrm{~W}\end{array}$ & $\begin{array}{ll}\text { sect ioris } & 12,13 \\
\text { sections } & 7,8,16,17,18,19,24 \\
& 22,27,28,29,33\end{array}$ & $\begin{array}{l}\text { Alperia } \\
\text { Green for est }\end{array}$ & Batesville Sandstone & $1,220-1,500$ & 59 \\
\hline 524 & $\mathrm{~T} 18 \mathrm{~N}$ & R24W & sectiuns 27,28 & Marble & Buulle formation & $1,440-1,700$ & 3 \\
\hline 525 & $\mathrm{~T} 18 \mathrm{~N}$ & $\mathrm{k} 23 \mathrm{~W}$ & sections 31,32 & Osage & Batesville Sandstone & $1,600-1,920$ & 3 \\
\hline 526 & $\mathrm{~T} 18 \mathrm{~N}$ & $\mathrm{R} 23 \mathrm{~W}$ & sections $26,34,35$ & Usage & Boone Formation & $1,320-1,560$ & 4 \\
\hline 527 & $118 \mathrm{~N}$ & $R 22 m$ & section 16 & Osage NE & Batesville Sandstone & 1.460 & 2 \\
\hline 528 & T17N & R23w & section 18 & Usage & Batesville Sandstone & $1,560-1,600$ & 2 \\
\hline 529 & $\mathrm{~T} 21 \mathrm{~N}$ & $\mathrm{k} 2 \mathrm{bW}$ & section $\angle b$ & Eureka Springs & Boone formation & 1,520 & 1 \\
\hline 530 & $121 N$ & R2bW & section $2 b$ & tureka Springs & Boone formation & 1,520 & 1 \\
\hline 531 & $\mathrm{I} 21 \mathrm{~N}$ & R26W & section 36 & Eureka Springs & Boone Formation & 1,200 & 1 \\
\hline 532 & T2ON & $R 2 b W$ & section 4 & Eureka Springs & Cotter Dolomite & 1.140 & 1 \\
\hline 533 & T $20 \mathrm{~N}$ & $\mathrm{R} 25 \mathrm{w}$ & section 8 & Eureka Springs & Cotter Dolomite & 1,140 & 1 \\
\hline
\end{tabular}


Table 8. - Identification of general areas of sinkholes and internally-drained. closed depressions identifiable on 7.5-minute topographic quadrangle maps in Carroll County. Arkansas--Continued

\begin{tabular}{|c|c|c|c|c|c|c|c|c|}
\hline $\begin{array}{c}\text { Site } \\
\text { number }\end{array}$ & Gene & erat & areas of & occurrence & $\begin{array}{l}71 / 2 \text { minute } \\
\text { topographic } \\
\text { quadrangle }\end{array}$ & $\begin{array}{l}\text { Geologic forma- } \\
\text { tion in which } \\
\text { depression occurs }\end{array}$ & $\begin{array}{l}\text { Altitude } \\
\text { of bottom of } \\
\text { depression } \\
\text { (feet above } \\
\text { sea level) }\end{array}$ & $\begin{array}{c}\text { Number } \\
\text { of } \\
\text { closed } \\
\text { depressions }\end{array}$ \\
\hline 534 & T2ON & R25W & section & 35 & Rockhouse & Cotter Dolomite & 1,160 & 1 \\
\hline 535 & T19N & R25W & section & 3 & Rockhouse & Boone formation & 1,660 & 1 \\
\hline 536 & $\mathrm{~T} 21 \mathrm{~N}$ & R24W & section & 36 & Blue Eye & Boune Formation & 1,340 & 1 \\
\hline 537 & T $21 \mathrm{~N}$ & $\mathrm{R} 23 \mathrm{~W}$ & section & 28 & Blue Eye & Boone Formation & 1,350 & 1 \\
\hline 538 & $\mathrm{~T} 21 \mathrm{~N}$ & $\mathrm{R} 22 \mathrm{~W}$ & section & 31 & Denver & Boone Formation & 1,140 & 1 \\
\hline 539 & $\mathrm{~T} 21 \mathrm{~N}$ & R22W & section & 29 & Denver & Boone Formation & 1,120 & 1 \\
\hline 540 & T2ON & $R 22 W$ & section & 6 & Denver & Boone Formation & 1,180 & 1 \\
\hline 541 & $\mathrm{~T} 20 \mathrm{~N}$ & $R 23 w$ & section & 3 & Blue Eye & Boone Formation & 1,140 & 1 \\
\hline 542 & T2ON & $\mathrm{R} 23 \mathrm{~W}$ & section & 25 & Green Forest & Boone Formation & 1,200 & 1 \\
\hline 543 & $\mathrm{~T} 2 \mathrm{ON}$ & $R 22 W$ & section & 31 & Alpena & Boone Formation & 1,160 & 1 \\
\hline 544 & T2ON & $\mathrm{R} 22 \mathrm{~W}$ & section & 16 & Denver & Hoone Formation & 1,120 & 1 \\
\hline 545 & T19N & $\mathrm{R} 23 \mathrm{~W}$ & section & 11 & Green Forest & Batesville Sandstone & 1,220 & 1 \\
\hline 546 & T19N & R23W & section & 17 & Green Forest & Boone formation & 1,560 & 1 \\
\hline 547 & T19N & R23W & section & 22 & Green Forest & Batesville Sandstone & 1,320 & 1 \\
\hline 548 & T 19N & R23W & section & 30 & Green forest & Cotter Dolomite & 1,350 & 1 \\
\hline 549 & $\mathrm{~T} 19 \mathrm{~N}$ & R23W & section & 27 & Green forest & Batesville Sandstone & 1,850 & 1 \\
\hline 550 & T 19N & $R 23 W$ & section & 32 & Green Forest & Cotter Dolomite & 1,300 & 1 \\
\hline 551 & T19N & R23W & section & 34 & Green forest & Boone formation & 1,900 & 1 \\
\hline 552 & T19N & $\mathrm{R} 22 \mathrm{w}$ & section & 31 & Alpena & Batesville Sandstone & 1,980 & 1 \\
\hline 553 & $\mathrm{~T} 18 \mathrm{~N}$ & R23W & section & 6 & Osage & Batesville Sandstone & 1,660 & 1 \\
\hline 554 & $\mathrm{~T} 18 \mathrm{~N}$ & $\mathrm{R} 23 \mathrm{~W}$ & section & 10 & Osage & Boone formation & 1,420 & 1 \\
\hline 555 & $\mathrm{~T} 18 \mathrm{~N}$ & $R 24 W$ & section & 13 & Osage & Boone Formation & 1,560 & 1 \\
\hline 556 & T 18N & R23W & section & 20 & Osage & Boone Formation & 1,800 & 1 \\
\hline 557 & $\mathrm{~T} 18 \mathrm{~N}$ & R23w & section & 23 & Osage & Batesville Sandstone & 1,720 & 1 \\
\hline 558 & T $18 \mathrm{~N}$ & $\mathrm{R} 22 \mathrm{~W}$ & section & 19 & Osage NE & Boone formation & 1,560 & 1 \\
\hline 559 & $\mathrm{~T} 17 \mathrm{~N}$ & $R 23 w$ & section & 9 & Osage & Atoka Formation & 2,000 & 1 \\
\hline 560 & $\mathrm{~T} 17 \mathrm{~N}$ & $\mathrm{R} 23 \mathrm{~W}$ & section & 6 & Osage & Boone Formation & 1,620 & 1 \\
\hline 561 & T17N & $\mathrm{R} 24 \mathrm{~W}$ & section & 12 & Osage & Batesville Sandstone & 1,700 & 1 \\
\hline 562 & $\mathrm{~T} 17 \mathrm{~N}$ & $R 24 W$ & section & 15 & Marble & Batesville Sandstone & 1,340 & 1 \\
\hline 563 & $T 18 N$ & R24W & section & 31 & Marble & Boune Formation & 1,360 & 1 \\
\hline 564 & T2ON & K23w & section & 33 & Green Forest & Batesville Sandstone & 1,360 & 1 \\
\hline 565 & T19N & $\mathrm{R} 22 \mathrm{~W}$ & section & 15 & Alpena & Boune formation & 1,280 & 1 \\
\hline 566 & $\mathrm{r} 19 \mathrm{~N}$ & $\mathrm{R} 22 \mathrm{~W}$ & section & 15 & Alpena & Boone formation & 1.180 & 1 \\
\hline 567 & I19N & $R 22 W$ & section & 21 & Alpena & Batesville Sandstone & 1.280 & 1 \\
\hline
\end{tabular}

